\title{
Limit properties of the monotone rearrangement for density and regression function estimation
}

\author{
DRAGI ANEVSKI ${ }^{1}$ and ANNE-LAURE FOUGÈRES ${ }^{2}$ \\ ${ }^{1}$ Centre for Mathematical Sciences, Lund University, BOX 118, 22100 Lund, Sweden. E-mail: dragi@ \\ maths.lth.se \\ ${ }^{2}$ Univ Lyon, Université Claude Bernard Lyon 1, CNRS UMR 5208, Institut Camille Jordan, 43 blvd. du 11 \\ novembre 1918, F-69622 Villeurbanne cedex, France. E-mail: fougeres@math.univ-lyon1.fr
}

The monotone rearrrangement algorithm was introduced by Hardy, Littlewood and Pólya as a sorting device for functions. Assuming that $x$ is a monotone function and that an estimate $x_{n}$ of $x$ is given, consider the monotone rearrangement $\hat{x}_{n}$ of $x_{n}$. This new estimator is shown to be uniformly consistent as soon as $x_{n}$ is. Under suitable assumptions, pointwise limit distribution results for $\hat{x}_{n}$ are obtained. The framework is general and allows for weakly dependent and long range dependent stationary data. Applications in monotone density and regression function estimation are detailed. Asymptotics for rearrangement estimators with vanishing derivatives are also obtained in these two contexts.

Keywords: density estimation; dependence; limit distributions; monotone rearrangement; regression function estimation

\section{Introduction}

Assume that $\left(t_{i}, x\left(t_{i}\right)\right)_{i=1}^{n}$, for some points $t_{i} \in[0,1]\left(\right.$ e.g. $\left.\left(t_{i}=i / n\right)\right)$, are pairs of data points. The (decreasing) sorting of the points $x\left(t_{i}\right)$ is then an elementary operation and produces the new sorted sequence of pairs $\left(t_{i}, y\left(t_{i}\right)\right)$ where $y=\operatorname{sort}(x)$ is the sorted vector. Let \# denote the counting measure of a set. Then we can define the sorting $y$ of $x$ by

$$
\begin{aligned}
& z(s)=\#\left\{t_{i}: x\left(t_{i}\right) \geq s\right\}, \\
& y(t)=z^{-1}(t),
\end{aligned}
$$

where $z^{-1}$ denotes the inverse of a function (if the points $x\left(t_{i}\right)$ are not unique it denotes the generalized inverse).

The "sorting" of a function $\{x(t), t \in[0,1]\}$ can then analogously be defined by the monotone rearrangement (cf. Hardy et al. [22]),

$$
\begin{aligned}
& z(s)=\lambda\{t \in[0,1]: x(t) \geq s\}, \\
& y(t)=z^{-1}(t),
\end{aligned}
$$


where the counting measure \# has been replaced by the Lebesgue measure $\lambda$, and $z^{-1}$ denotes the generalized inverse.

The monotone rearrangement algorithm of a set or a function has mainly been used as a device in analysis, see, for example, Lieb and Loss [24], Chapter 3, or in optimal transportation (see Villani [39], Chapter 3). Fougères [16] was the first to use the algorithm in a statistical context, for density estimation under order restrictions. Meanwhile, Polonik [30,31] also developed tools of a similar kind for density estimation for multivariate data. More recently, several authors revisited the monotone rearrangement procedure in the estimation context under monotonicity; see Dette et al. [13], Neumeyer [29], Chernozhukov et al. [10], Birke and Dette [6], Jankowski and Wellner [23], Volgushev and Dette [42], Birke et al. [7], Volguchev [40]. Some tests of monotonicity have also been recently introduced, see, for example, Volguchev et al. [41] and Birke et al. [8].

We introduce the following two-step approach for estimating a monotone function. Assume that $x$ is a monotone function on an interval $I \subset \mathbb{R}$. Assume also that we already have an estimate $x_{n}$ of $x$, but that this estimate is not necessarily monotone. The procedure adopted in this paper is to use the monotone rearrangement $\hat{x}_{n}$ of $x_{n}$ as an estimate of $x$.

Under the assumption that we have process limit distribution results for (a localized version of) the stochastic part of $x_{n}$ and that the deterministic part of $x_{n}$ is asymptotically differentiable at a fixed point $t_{0}$, with strictly negative derivative, we obtain pointwise limit distribution results for $\hat{x}_{n}\left(t_{0}\right)$. The framework is general and allows for weakly dependent as well as long range dependent data. This is the topic for Section 3, where we also explore in more detail the applications of our general results to monotone density and regression function estimation. These are the problems of estimating $f$ and $m$ respectively in

(i) $t_{1}, \ldots, t_{n}$ stationary observations with marginal decreasing density $f$ on $\mathbb{R}^{+}$,

(ii) $\left(t_{i}, y_{i}\right)$ observations from $y_{i}=m\left(t_{i}\right)+\varepsilon_{i}, t_{i}=i / n, i=1, \ldots, n, m$ decreasing on $[0,1]$, $\left\{\varepsilon_{i}\right\}$ stationary sequence with mean zero.

The standard approaches in these two problems have been isotonic regression for the regression problem, first studied by Brunk [9], and (nonparametric) Maximum Likelihood estimation (NPMLE) for the density estimation problem, first introduced by Grenander [19]. A wide literature exists for regression and density estimation under order restrictions. One can refer for example, to Mukerjee [28], Ramsay [33], Mammen [25], Hall and Huang [20], Mammen et al. [26], Gijbels [18], Birke and Dette [5], Dette and Pilz [14], Dette et al. [13] for the regression context. Besides, see Eggermont and Lariccia [15], Fougères [16], Hall and Kang [21], Meyer and Woodroofe [27], Polonik [30], Van der Vaart and Van der Laan [38], among others, for a focus on monotone (or unimodal) density estimation. Anevski and Hössjer [2] gave a general approach unifying both contexts. In their introduction, Birke and Dette [6] provide nice references in which physical or economical arguments justify the assumption of monotonicity. Our approach is similar in spirit to the general methods studied in Anevski and Hössjer [2] and first introduced in the regression estimation setting by Mammen [25]: Start with a preliminary estimator and make it monotone by projecting it on the space of monotone functions. The present approach can however at some point be considered preferable: The monotone rearrangement, being basically a sorting, is a simpler procedure than an $\mathbb{L}^{2}$-projection. Furthermore, the consistency and limit distribution results indicate similar properties to Mammen's and Anevski and Hössjer's estimators. Besides, an important advantage of our estimator is the finite sample behavior: Mammen's estimator is monotone but not necessarily smooth; Mammen actually studied 
two approaches, one with kernel smoothing followed by monotonization and the other approach the other way around, that is, monotonization followed by kernel smoothing. Mammen showed that the two proposals are first-order equivalent. However, their finite sample size properties are very different: the first resulting estimator is monotone but not necessarily smooth, while the other is smooth but not necessarily monotone, so that one needs to choose which property is more important. This is not the case with our estimator, since if we start with a smooth estimator of the function, for example, a kernel estimator, the monotone rearrangement will be smooth as well. This can however become a disadvantage for instance, when the estimand is discontinuous: then the monotone rearrangement will "oversmooth" since it will give a continuous result, while Mammen's estimator will keep more of the discontinuity intact.

Note that our results are geared towards local estimates, that is, estimates that use only a subset of the data and that are usually estimators of estimands that can be expressed as non-differentiable maps of the distribution function such as for example, density functions, regression functions, or spectral density functions. This differs from global estimates, as those considered for example, by Chernozhukov et al. [11] for quantile estimation. Chernozhukov et al. [11] rearrange the empirical quantile function, and use the fact that the rearrangement map is Hadamard differentiable together with Donsker type results, to obtain general statements about the final estimator. This approach is however not applicable in our case. In fact, our preliminary estimators are all local estimators, and they do not converge weakly as processes. Therefore the Hadamard differentiability of $T$ has no implication in our estimation problems; we need to make a more detailed reasoning, assuming local limit process results for the preliminary estimator, together with properties of the map T. These two features may be seen as replacements for the Donsker result and the Hadamard differentiability result of $T$, that are used in [11].

An approach similar to ours for local estimates is given in Dette et al. [13], using a modified version of the Hardy-Littlewood-Pólya monotone rearrangement: The first step consists of calculating the upper level set function and is identical to ours. However, in the second step they use a smoothed version of the (generalized) inverse, which avoids nonregularity problems for the inverse map. The resulting estimator is therefore not rate-optimal, and the limit distributions are standard Gaussian due to the oversmoothing.

Using kernel estimators as preliminary estimators of $f$ and $m$ on which the monotone rearrangement is then applied, we are able to derive limit distribution results for quite general dependence situations, demanding essentially stationarity for the underlying random parts $\left\{t_{i}\right\}$ and $\left\{\varepsilon_{i}\right\}$, respectively. The results are however stated in a form that allows for other estimators than the kernel based as starting points, for example, wavelet or splines estimators.

The paper is organized as follows: In Section 2, we present the monotone rearrangement algorithm as classically defined, and we derive some simple properties that will be used in the sequel. Then we define the generic estimator of the monotone function of interest, and state the consistency for the estimator.

In Section 3, the pointwise limit distribution properties are considered. For this purpose, we need to generalise the monotone rearrangement map for some specific functions, as will be done in Sections 3.1 and 3.2. The limit distribution given in Theorem 4 is of the general form

$$
d_{n}^{-1}\left[\hat{x}_{n}\left(t_{0}\right)-x\left(t_{0}\right)\right] \stackrel{\mathcal{L}}{\rightarrow} T\left(A \cdot+\tilde{v}\left(\cdot ; t_{0}\right)\right)(0)+\Delta,
$$


where $T$ is the monotone rearrangement map;

$$
\Delta=\lim _{n \rightarrow \infty} d_{n}^{-1}\left[\mathbb{E}\left\{x_{n}\left(t_{0}+s d_{n}\right)\right\}-x\left(t_{0}\right)\right]
$$

is the asymptotic local bias of the preliminary estimator; $A$ is the uniform limit, in $s$ over compact intervals,

$$
d_{n}^{-1}\left\{x_{b, n}\left(t_{0}+s d_{n}\right)-x_{b, n}\left(t_{0}\right)\right\} \rightarrow A
$$

(typically with $A=x^{\prime}\left(t_{0}\right)$ in our applications); and

$$
\tilde{v}\left(s ; t_{0}\right) \stackrel{\mathcal{L}}{=} \lim _{n \rightarrow \infty} d_{n}^{-1}\left[x_{n}\left(t_{0}+s d_{n}\right)-\mathbb{E}\left\{x_{n}\left(t_{0}+s d_{n}\right)\right\}\right]
$$

is the weak local limit of the process part of the preliminary estimator; here $d_{n} \downarrow 0$ is a deterministic sequence that is determined by the dependence structure of the data. We then apply the obtained results to regression function estimation and density estimation under order restrictions, and derive the limit distributions for the estimators. This gives rise to some new universal limit random variables, such as for example, in the regression context $T(s+B(s))(0)$ with $T$ the monotone rearrangement map and $B$ standard two sided Brownian motion for independent and weakly dependent data, or $T\left(s+B_{1, \beta}(s)\right)(0)$ with $B_{1, \beta}$ fractional Brownian motion with self similarity parameter $\beta$, when data are long range dependent. The rate of convergence $d_{n}$ is e.g. for the regression problem the optimal $n^{-1 / 3}$ in the i.i.d. and weakly dependent data context and of a non-polynomial rate in the long range dependent context, similarly to previously obtained results in isotonic regression for long range dependent data, cf. Anevski and Hössjer [2].

In Section 4, we derive limit distribution results for the proposed estimator in the case when the estimand has $q$ vanishing derivatives while its $(q+1)$ st derivative is strictly negative. The limit results are given in a general setting, and applied to both the density function and regression function estimation cases, and in similar dependence settings as for the "regular" case, that are derived in Sections 3.3 and 3.4. The limit distribution results are now of the form

$$
d_{n}^{-1}\left[\hat{x}_{n}\left(t_{0}\right)-x\left(t_{0}\right)\right] \stackrel{\mathcal{L}}{\rightarrow} T\left(A_{x}(\cdot)+\tilde{v}\left(\cdot ; t_{0}\right)\right)(0),
$$

where $T$ is the monotone rearrangement map, $A_{x}(s)$ is a function that is given as a uniform limit over compact intervals,

$$
d_{n}^{-(q+1)}\left(x_{b, n}\left(t_{0}+s d_{n}\right)-x\left(t_{0}\right)\right) \rightarrow A_{x}(s)
$$

as $d_{n} \rightarrow 0$, which (for symmetric kernels) is a convolution of a degree $q+1$ monomial with the kernel, while $\tilde{v}$ is the limit process that turns up in the above. The rate $d_{n}$ is now different: It is slower than above, and for example, for independent data, in both the density estimation and regression function estimation contexts, it is $d_{n}=n^{-1 /(3+2 q)}$.

In Appendix A, we give some proofs for the results of Section 2. In Appendix B, we state a general result on maximal bounds on the rescaled process part. The proof of this result is 
provided in Section 1 of the supplemental article (Anevski and Fougères [1]). Therein, further useful but technical results on maximal bounds on the rescaled process parts in the density and regression estimation problems are also derived, that is, for the local partial sum process and empirical processes, for weakly dependent as well as long range dependent data. Furthermore, in Section 2 of the supplemental article (Anevski and Fougères [1]), we present a simulation study that illustrates the finite sample behaviour of our estimator, and compare it to other estimators that are considered in the paper of Birke and Dette [6].

In this context, it may be instructive to compare our results with previously obtained results, for similar procedures. The estimator defined by Dette et al. [13], is a two step procedure similar to ours for regression estimation problems, consisting of first defining a smooth estimate of the estimand and next do the monotone rearrangement of that estimator. We would like to point out that the assumptions in the two approaches are somewhat different: In our paper, we use a fixed design setting, which enables us to use the Gasser-Müller estimator as the first step estimator, while the results in Dette et al. [13] are derived in a random design setting. In [13], is used however an extra smoothing procedure in the second step, and therefore their obtained estimator is not the same as ours, and in fact their estimator may be seen as a smooth monotone rearrangement of the preliminary estimator. More interestingly, the two estimators give qualitatively very different results, with different rates and different limit random variables. Within the class of continuously differentiable monotone functions, the estimator considered in [13] is not rate optimal (for the independent data case, which is the only case they consider), they get a slower rate than the optimal, cf. Theorem 3.2 in [13]. Furthermore, their limit random variable is Gaussian, with the Gaussian distribution turning up due to the over smoothing in the second extra step, whereas ours converge to the above defined new universal random variable. We would like to also emphasize that we are able to state our results for also dependent data, covering both weak and strong dependence. In Neumeyer [29], the same estimator as ours is treated, for general estimands and thus treating both regression and density estimation problem; the consistency of the resulting estimator is derived (see Neumeyer [29], Theorem 3.1).

\section{The monotone estimation procedure}

\subsection{Monotone rearrangement: First definitions}

Monotone rearrangements were originally defined by Hardy et al. [22], Chapter 10.12, for nonnegative and integrable functions on [0,1]. In Lieb and Loss [24], Chapter 3, the definition is extended to Borel measurable functions from $\mathbb{R}^{n}$ into $\mathbb{C}$ that vanish at infinity. We use their definition for Borel measurable functions from $\mathbb{R}$ into $\mathbb{R}^{+}$that vanish at infinity, in the sense that for each positive $u$

$$
r_{f}(u):=\lambda\{t \in \mathbb{R}: f(t)>u\}<+\infty,
$$

where $\lambda(A)$ denotes the Lebesgue measure of any Borel set $A$ on $\mathbb{R}$. Note that this definition holds in particular for integrable functions like densities on $\mathbb{R}^{+}$as considered in Fougères [16]. 
Definition 1. Let $f$ be a positive function defined on $\mathbb{R}^{+}$satisfying (1). The monotone rearrangement of $f$ is defined as the (right continuous) generalized inverse of $r_{f}$, namely

$$
T(f)(t):=\inf \left\{u \in \mathbb{R}^{+}: r_{f}(u) \leq t\right\},
$$

for each positive $t$.

This rearrangement satisfies several properties that will be listed later in this section, see Lemmas 2 and 3 and Theorem 1. Note that related results were obtained by Chernozhukov et al. [11].

A particular class of functions for which (1) is satisfied is the set of bounded functions defined on a finite interval $I \subset \mathbb{R}$. Denote $\mathcal{B}(I)=\{f: f(I)$ bounded $\}$ and $\mathcal{D}(I)=\{f$ : $f$ decreasing on $I$ \}. Let $r_{f, I}$ be the right continuous map from $f(I)$ to $\mathbb{R}^{+}$, defined for each $u \in f(I)$ by

$$
r_{f, I}(u):=\lambda\{t \in I: f(t)>u\}=\lambda\left\{I \cap f^{-1}(u, \infty)\right\}
$$

as the (right continuous) generalized inverse of $r_{f, I}$

$$
T_{I}(f)(t):=\inf \left\{u \in f(I): r_{f, I}(u) \leq t-\inf I\right\} .
$$

The following lemmas and theorem are listing some simple and useful properties of the maps $u \mapsto r_{f, I}(u), f \mapsto r_{f, I}$ and $f \mapsto T_{I}(f)$ respectively. The proofs are straightforward and relegated to Appendix A for more clarity.

Lemma 1. Assume $I \subset \mathbb{R}$ is a finite interval, and $f \in \mathcal{B}(I)$. Then

(i) If $f$ has no flat regions on $I$, that is, $\lambda\left\{I \cap f^{-1}(\{u\})\right\}=0$ for all $u \in f(I)$, then $r_{f, I}$ is continuous,

(ii) If there is a $u_{0} \in f(I)$ such that $\lambda\left\{I \cap f^{-1}\left(\left\{u_{0}\right\}\right)\right\}=c>0$, then $r_{f, I}$ has a discontinuity at $u_{0}$ of height $c$,

(iii) If $f$ has a discontinuity at $t_{0} \in I$ and $f$ is decreasing, then $r_{f, I}$ admits a flat region with level $t_{0}$.

Lemma 2. Let $I \subset \mathbb{R}$ be a finite interval, and assume $f \in \mathcal{B}(I)$. Then

(i) If $c$ is a constant then $r_{f+c, I}(u)=r_{f, I}(u-c)$, for each $u \in f(I)+c$.

(ii) $r_{c f, I}(u)=r_{f, I}(u / c)$ if $c>0$, for each $u \in c f(I)$.

(iii) $f \leq g \Rightarrow r_{f, I} \leq r_{g, I}$.

(iv) Let $f_{c}(t)=f(t c)$. Then $\mathrm{cr}_{f_{c}, I}=r_{f, I}$.

(v) Let $f_{c}(t)=f(t+c)$. Then $r_{f_{c}, I}=r_{f, I}$.

Lemma 3. Let $I \subset \mathbb{R}$ be a finite interval and assume $f, g$ are functions in $\mathcal{B}(I)$. The monotone rearrangement map $T_{I}$ satisfies the following:

(i) $T_{I}(f+c)=T_{I}(f)+c$, if $c$ is a constant;

(ii) $T_{I}(c f)=c T_{I}(f)$, if $c>0$ is a constant; 
(iii) $f \leq g \Rightarrow T_{I}(f) \leq T_{I}(g)$;

(iv) Let $f_{c}(t)=f(c t)$; then $T_{I / c}\left(f_{c}\right)(t)=T_{I}(f)(c t)$;

(v) Let $f_{c}(t)=f(t+c)$; then $T_{I-c}\left(f_{c}\right)(t)=T_{I}(f)(t+c)$.

The previous result implies that the map $T_{I}$ is continuous, as stated in the following theorem.

Theorem 1. Let $\|\cdot\|$ be the supremum norm on $\mathcal{B}(I)$. Then the map $T_{I}$ is a contraction, i.e. $\left\|T_{I}(f)-T_{I}(g)\right\| \leq\|f-g\|$. In particular, $T_{I}$ is a continuous map, that is, for all $f_{n}, f \in \mathcal{B}(I)$,

$$
\left\|f_{n}-f\right\| \rightarrow 0 \Rightarrow\left\|T_{I}\left(f_{n}\right)-T_{I}(f)\right\| \rightarrow 0,
$$

as $n$ tends to infinity.

Note that Lemma 2 holds (with identical proof) for $T$ as defined in (2), and Lemma 3 follows from that. Thus Theorem 1 also holds in this case for the supremum norm over $\mathbb{R}^{+}$, with identical proof.

Remark 1. One can also refer to Lieb and Loss [24], Theorem 3.5, for a proof of the contraction property (the "non expansivity" property of the map $T_{I}$ ), for the $L^{p}$-norms for functions $f$ and $g$ vanishing at infinity.

Finally, observe that when the function $f$ is replaced by a stochastic process $x$ defined almost surely, then for almost every realisation of $x$, one can define $r_{x}$ (resp. $r_{x, I}$ ) and thereafter its generalized inverse. Thus one can define the monotone rearrangement almost surely for every stochastic process with finite support or satisfying (1) almost surely. We will make use of this last concept to define new estimators in the next section.

\subsection{The new estimators: Definition and first properties}

Let $x$ be a function of interest such as a density function, a regression function, or a spectral density, for example. Assume $x$ is non increasing. Consider an estimator $x_{n}$ of $x$ constructed from $n$ observations, which is not supposed to be monotone. Typically, $x_{n}$ can be an estimator based on kernel, wavelets, splines, etc. Let $\aleph_{n}$ denote the support of $x_{n}$. Assume that $x_{n}$ is such that it is possible to define either $T\left(x_{n}\right)$ as in (2) (when $\aleph_{n}$ is infinite) or $T_{\aleph_{n}}\left(x_{n}\right)$ as in (3) (when $\aleph_{n}$ is finite). This will in particular be the case as soon as $x_{n}$ is a density of $\mathcal{B}\left(\mathbb{R}^{+}\right)$, or $x_{n}$ is a regression function on $[0,1]$. For sake of simplicity, a unique notation $T$ will be used in the following to refer equally to $T$ or $T_{\aleph_{n}}$.

Definition 2. We define as a new estimator of $x$ the monotone rearrangement of $x_{n}$, namely $T\left(x_{n}\right)$. This is a nonincreasing estimator of $x$.

Theorem 2. (i) Assume that $\left\{x_{n}\right\}_{n \geq 1}$ is a uniformly consistent estimator of $x$ (in probability, uniformly on a compact set $B \subset \mathbb{R})$. If $x$ is nonincreasing, then $\left\{T\left(x_{n}\right)\right\}_{n \geq 1}$ is also a uniformly consistent estimator of $x$ (in probability, uniformly on $B$ ). 
(ii) Assume that $\left\{x_{n}\right\}_{n \geq 1}$ is an estimator that converges in probability in $\mathbb{L}^{p}$-norm to $x$, and that $\left\{x_{n}\right\}_{n \geq 1}$ and $x$ are vanishing at infinity. If $x$ is nonincreasing, then $\left\{T\left(x_{n}\right)\right\}_{n \geq 1}$ also converges in probability in $\mathbb{L}^{p}$-norm to $x$.

Proof. Part (i) follows from the fact that $\|x\|=\sup _{t \in K}|x(t)|$ is a norm (for every compact $K \subset \mathbb{R}$ ), that $T(x)=x$ if $x$ is non increasing, and that $T$ is a contraction with respect to $\|\cdot\|$, by Theorem 1. To get (ii), assume that $\left\|x_{n}-x\right\|_{L^{p}} \stackrel{P}{\rightarrow} 0$, and then note that

$$
\left\|T\left(x_{n}\right)-x\right\|_{L^{p}}=\left\|T\left(x_{n}\right)-T(x)\right\|_{L^{p}} \leq\left\|x_{n}-x\right\|_{L^{p}},
$$

thanks to Lieb and Loss [24], Theorem 3.5.

Remark 2. The strong convergence in $\mathbb{L}^{p}$-norm of $T\left(f_{n}\right)$ to $f$, as a consequence of the corresponding result for $f_{n}$, was first established in Fougères [16], Theorem 5, in the case when $f_{n}$ is the kernel estimator of a density function $f$; the assumption that the functions $\left\{f_{n}\right\}, f$ vanish at infinity are then naturally satisfied. Chernozhukov et al. [10] give a refinement of the non expansivity property, see their Proposition 1, part 2, providing a bound for the gain done by rearranging $f_{n}$ and examining the multivariate framework as well.

\section{Limit distribution results}

Simple monotone estimators have been defined in Section 2.2 that satisfy several desired consistency properties. The aim of this section is to go further into asymptotic properties, focusing on pointwise limit distribution results for these estimators. To this purpose, we will first provide a more general definition of monotone rearrangement for some specific functions (and processes), and then state in Section 3.2 the main result of the paper in a general setting. The particular results obtained for density regression function estimations will next be developed.

\subsection{Extension of monotone rearrangement algorithm}

If $\varphi$ is a function for which $r_{\varphi}(u)$ is possibly infinite for some positive $u$, a definition of $T(\varphi)$ can be given locally around a fixed point $x \in I_{0}$, where $I_{0}$ is a finite interval, as soon as the function $\varphi$ satisfies the following property:

Let $I_{0}$ and the function $\varphi$ be given. Assume there exists a constant $M=M\left(\varphi, I_{0}\right)<\infty$ and a finite interval $I_{1}=I_{1}\left(\varphi, I_{0}\right) \supset I_{0}$ such that

$$
\begin{aligned}
& \inf _{t \in\left(\inf I_{1}, \sup I_{0}\right)} \varphi(t)>-M \quad \text { and } \sup _{t \in\left(\sup I_{1}, \infty\right)} \varphi(t)<-M, \\
& \inf _{t \in\left(-\infty, \inf I_{1}\right)} \varphi(t)>+M \quad \text { and } \sup _{t \in\left(\inf I_{0}, \sup I_{1}\right)} \varphi(t)<+M .
\end{aligned}
$$

Theorem 3. Let $I_{0}$ be a finite and fixed interval, and let $\varphi$ be a continuous function $\mathbb{R}$ such that (4) and (5) are satisfied. Then for any finite interval $J$ containing $I_{1}$, one has $T_{J}(\varphi) \equiv T_{I_{1}}(\varphi)$ on $I_{0}$. 
Proof. The proof consists of the following three steps: (i) We construct a point $y_{*}$ on which $T_{J}$ and $T_{I_{1}}$ agree, that is, such that $T_{J}(\varphi)\left(y_{*}\right)=T_{I_{1}}(\varphi)\left(y_{*}\right)$. (ii) We show that inf $I_{1} \leq y_{*} \leq \inf I_{0}$. (iii) We show that if $T_{J}(\varphi)$ and $T_{I_{1}}(\varphi)$ agree at $y_{*}$, they will coincide on $\left[y_{*}, \sup I_{o}\right]=: \overline{\tilde{I}}$.

(i) Define $y_{1}:=\inf \left\{y \in I_{1}: \varphi(y)<\varphi([\inf J, y))\right\}$. Then by (5) we have that $\varphi\left(y_{1}\right) \geq+M$, which by the definition of $y_{1}$ implies that also inf $\varphi\left(\left[\inf J, y_{1}\right)\right) \geq+M$. Furthermore, from the left part of (4) and the right part of (5) it follows that $\varphi\left(I_{0}\right) \subset(-M,+M)$. This implies that

$$
\begin{aligned}
y_{1} & <\inf \{y \in J: \varphi(y) \in(-M, M)\} \\
& \leq \inf \left\{y \in J: \varphi(y) \in \varphi\left(I_{0}\right)\right\} \\
& =: z_{0},
\end{aligned}
$$

where the first inequality follows from continuity of $\varphi$, the definition of $y_{1}$ and the theorem of intermediate values. Thus $y_{1} \in I_{1}, z_{0} \leq \inf I_{0}$ and $y_{1}<z_{0}$ and so also $z_{0} \in I_{1}$.

As a consequence, one has

$$
\begin{aligned}
r_{\varphi, J}\left\{\varphi\left(y_{1}\right)\right\}= & \lambda\left\{t \in J: \varphi(t)>\varphi\left(y_{1}\right)\right\} \\
= & \lambda\left\{t \in J \cap\left(-\infty, y_{1}\right): \varphi(t)>\varphi\left(y_{1}\right)\right\} \\
& +\lambda\left\{t \in J \cap\left(y_{1}, \infty\right): \varphi(t)>\varphi\left(y_{1}\right)\right\} \\
= & y_{1}-\inf J+\lambda\left\{t \in I_{1} \cap\left(y_{1}, \infty\right): \varphi(t)>\varphi\left(y_{1}\right)\right\},
\end{aligned}
$$

where the first two terms in the last equality follow since from the definition of $y_{1}, \varphi(t)>\varphi(y)$ for all $t \in J \cap\left(y_{1}, \infty\right)$, while the last term in the last equality follows from $y_{1} \in I_{1}$. Similarly, one has

$$
\begin{aligned}
r_{\varphi, I_{1}}\left\{\varphi\left(y_{1}\right)\right\}= & \lambda\left\{t \in I_{1} \cap\left(-\infty, y_{1}\right): \varphi(t)>\varphi\left(y_{1}\right)\right\} \\
& +\lambda\left\{t \in I_{1} \cap\left(y_{1}, \infty\right): \varphi(t)>\varphi\left(y_{1}\right)\right\} \\
= & y_{1}-\inf I_{1}+\lambda\left\{t \in I_{1} \cap\left(y_{1}, \infty\right): \varphi(t)>\varphi\left(y_{1}\right)\right\}
\end{aligned}
$$

Thus the following equality holds

$$
r_{\varphi, J}\left\{\varphi\left(y_{1}\right)\right\}+\inf J=r_{\varphi, I_{1}}\left\{\varphi\left(y_{1}\right)\right\}+\inf I_{1}:=y_{\star} .
$$

Then $T_{J}(\varphi)\left(y_{\star}\right)=\inf \left\{u \in \varphi(J): r_{\varphi, J}(u) \leq r_{\varphi, J}\left(\varphi\left(y_{1}\right)\right)\right\}$, which since $r_{\varphi, J}$ is a decreasing function, is equal to $\varphi\left(y_{1}\right)$. Similarly, $\varphi\left(y_{1}\right)=T_{I_{1}}(\varphi)\left(y_{\star}\right)$, and we have shown that $T_{J}(\varphi)\left(y_{\star}\right)=$ $\varphi\left(y_{1}\right)=T_{I_{1}}(\varphi)\left(y_{\star}\right)$, and thus the two maps agree at $y_{\star}$.

(ii) From the right-hand parts of (4) and (5) follow that

$$
T_{J}(\varphi)\left(\inf I_{0}\right) \leq M .
$$

Furthermore,

$$
T_{J}(\varphi)\left(y_{*}\right)=\varphi\left(y_{1}\right) \geq M .
$$


Since $T_{J}(\varphi)$ is a decreasing function (7) and (8) imply that $y_{*} \leq \inf I_{0}$, and (6) implies that $y_{*} \leq \inf I_{1}$.

(iii) Finally, we prove that if $T_{J}(\varphi)$ and $T_{I_{1}}(\varphi)$ coincide at $y_{\star}$, they will coincide on $\tilde{I}=$ $\left[y_{*}, \sup I_{0}\right]$. Let $u \in\left[-M, \varphi\left(y_{1}\right)\right]=: \tilde{Y}$ be arbitrary, and write on one hand

$$
\begin{aligned}
r_{\varphi, J}(u)= & \lambda\{t \in J: \varphi(t)>u\} \\
= & \lambda\left\{t \in J: \varphi(t)>\varphi\left(y_{1}\right)\right\}+\lambda\left\{t \in J: \varphi\left(y_{1}\right) \geq \varphi(t)>u\right\} \\
= & r_{\varphi, J}\left\{\varphi\left(y_{1}\right)\right\}+\lambda\left\{t \in J \cap\left(y_{1}, \infty\right): \varphi\left(y_{1}\right) \geq \varphi(t)>u\right\} \\
= & r_{\varphi, I_{1}}\left\{\varphi\left(y_{1}\right)\right\}+\inf I_{1}-\inf J \\
& +\lambda\left\{t \in I_{1} \cap\left(y_{1}, \infty\right): \varphi\left(y_{1}\right) \geq \varphi(t)>u\right\},
\end{aligned}
$$

where the next to last equality follows since by the definition of $y_{1}, \varphi(t) \leq \varphi\left(y_{1}\right)$ only for $t>y_{1}$, and the last equality follows from (6) and since $y_{1} \in I_{1}$. On the other hand,

$$
\begin{aligned}
r_{\varphi, I_{1}}(u) & =r_{\varphi, I_{1}}\left\{\varphi\left(y_{1}\right)\right\}+\lambda\left\{t \in I_{1}: \varphi\left(y_{1}\right) \geq \varphi(t)>u\right\} \\
& =r_{\varphi, I_{1}}\left\{\varphi\left(y_{1}\right)\right\}+\lambda\left\{t \in I_{1} \cap\left(y_{1}, \infty\right): \varphi\left(y_{1}\right) \geq \varphi(t)>u\right\},
\end{aligned}
$$

so that

$$
r_{\varphi, J}(u)+\inf J=r_{\varphi, I_{1}}(u)+\inf I_{1} .
$$

Next, since $T_{J}(\varphi)$ and $T_{I_{1}}(\varphi)$ are decreasing, $T_{J}(\varphi)\left(y_{*}\right)=T_{I_{1}}(\varphi)\left(y_{*}\right)=\varphi\left(y_{1}\right)$, inf $I_{1} \leq y_{*} \leq$ $\inf I_{0}$, and $\inf \varphi\left(\left[y_{\star}, \sup I_{0}\right]\right)>-M$, we obtain

$$
\begin{aligned}
& T_{J}(\varphi)(\tilde{I}) \subset \tilde{Y}, \\
& T_{I_{1}}(\varphi)(\tilde{I}) \subset \tilde{Y} .
\end{aligned}
$$

Therefore, for $t \in \tilde{I}$,

$$
\begin{aligned}
T_{J}(\varphi)(t) & =\inf \left\{u \in \varphi(J): r_{\varphi, J}(u) \leq t-\inf J\right\} \\
& =\inf \left\{u \in \tilde{Y}: r_{\varphi, J}(u) \leq t-\inf J\right\} \\
& =\inf \left\{u \in \tilde{Y}: r_{\varphi, I_{1}}(u) \leq t-\inf I_{1}\right\} \\
& =\inf \left\{u \in \varphi\left(I_{1}\right): r_{\varphi, I_{1}}(u) \leq t-\inf I_{1}\right\} \\
& =T_{I_{1}}(\varphi)(t) .
\end{aligned}
$$

The second equality above holds since $T_{J}(\varphi)\left(y_{*}\right)=\varphi\left(y_{1}\right)=\sup \tilde{Y}$, inf $\tilde{I}=y_{*}, T_{J}(\varphi)$ is decreasing and because of the first part of (10), the third equality follows from (9), and the next to last equality is similar to the second (with $T_{I_{1}}$ replacing $T_{J}$ and using the second part of (10)).

Note also that the interval $I_{1}$ can without loss of generality be taken to be symmetric around 0 e.g. as $I_{1}=[-k, k]$ : In fact assuming that (4) and (5) hold with some $I_{1}$ and $M$ we can replace 
$I_{1}$ with $[-k, k]$, with $k=\max \left(\left|\inf I_{1}\right|,\left|\sup I_{1}\right|\right)$, then (4) and (5) will hold for $I_{1}=[-k, k]$ and with the same $M$.

Corollary 1. Let $I_{0} \subset \mathbb{R}$ be a finite and fixed interval. Assume $\varphi$ is continuous and satisfies (4) and (5). Then for each $t \in I_{0}$, one can define

$$
T(\varphi)(t):=\lim _{k \rightarrow+\infty} T_{[-k, k]}(\varphi)(t)
$$

Remark 3. Even if this definition seems to be dependent on $I_{1}=I_{1}\left(\varphi, I_{0}\right)$, it is not, because of Theorem 3 and since we define $T(\varphi)$ locally, namely only on $I_{0}$. So in particular one has $T(\varphi)(t)=T_{I_{1}}(t)$ for each $t \in I_{0}$.

We next state a simple condition that ensures (4) and (5).

Lemma 4. Let $\varphi$ be a locally bounded function on $\mathbb{R}$, such that

$$
\lim _{x \rightarrow-\infty} \varphi(x)=-\lim _{x \rightarrow+\infty} \varphi(x)=+\infty
$$

Then for any interval $I_{0}$ there exists a finite interval $I_{1} \supset I_{0}$ and a finite constant $M$ such that (4) and (5) hold.

Proof. Let $I_{0}=[a, b]$ and put $M=\sup _{x \geq a} \varphi(x)$. Since $\varphi$ is locally bounded and $\lim _{x \rightarrow+\infty} \varphi(x)=-\infty$ it follows that $M<\infty$. Since $\lim _{x \rightarrow-\infty} \varphi(x)=+\infty$ there is a $c<a$ such that $\varphi(x)>M$ for all $x \leq c$. Let $c_{M}:=\sup \{x<a: \varphi(t)>M \forall t \leq x\}$. Define then $m=\inf _{x \in\left[c_{M}, b\right]} \varphi(x)$. Since $\lim _{x \rightarrow \infty} \varphi(x)=-\infty$, there exists a $d>b$ such that for all $x \geq d$, $\varphi(x)<\min (m,-M)$. Let $d_{m, M}:=\inf \{x>b: \varphi(t)<\min (m,-M) \forall t>x\}$. Then (4) and (5) hold with $I_{1}=\left[c_{M}, d_{m, M}\right]$.

Note that $T$ as defined in (2) is a continuous map with the metric generated by the supnorm on compact intervals, while $T$ defined as a extension via the local definition in (11) is not. The first statement follows from the fact that $T$ as defined in (2) is easily seen to satisfy the properties in Theorem 1, with the supnorm over $I$ replaced by the the supnorm metric on compact intervals. The lack of continuity with the respect of uniform convergence on compact intervals is however of no importance for us, in our use of continuity for deriving consistency and limit distributions: We will derive limit distribution results only via local versions $T_{[-c, c]}$, for which we have established continuity in Theorem 1 . The consistency is derived using the global map only in the density estimation problem, for which we use definition (2) for $T$. For the regression problem, we apply our results to functions defined on $[0,1]$ and thus there is no need for a global definition then.

\subsection{Asymptotic distribution in a general framework}

Let $J \subset \mathbb{R}$ be a finite or infinite interval, and $C(J)$ the set of continuous functions on $J$. Let $\left\{x_{n}\right\}_{n \geq 1}$ be a sequence of stochastic processes in $C(J)$ and let $t_{0}$ be a fixed interior point in $J$. 
Assume that either $J$ is finite or that $x_{n}$ satisfies (1) almost surely, so that $T\left(x_{n}\right)$ the monotone rearrangement of $x_{n}$ can be defined almost surely, as seen in Section 2.1. In this section, limit distribution results for the random variable $T\left(x_{n}\right)\left(t_{0}\right)$ will be derived, where $T$ is the monotone rearrangement map defined as $T_{J}$ if $J$ is finite or $T$ if $J$ is infinite. The proof of these results are along the lines of Anevski and Hössjer [2], and their notation will be used for clarity. Decompose in particular $x_{n}$ into a deterministic part and a stochastic part

$$
x_{n}(t)=x_{b, n}(t)+v_{n}(t),
$$

for $t \in J$. Given a sequence $d_{n} \downarrow 0$ and an interior point $t_{0}$ in $J$ define $J_{n, t_{0}}=d_{n}^{-1}\left(J-t_{0}\right)$. Then, for $s \in J_{n, t_{0}}$, it is possible to rescale respectively the stochastic and deterministic parts of $x_{n}$ as

$$
\begin{aligned}
\tilde{w}_{n}\left(s ; t_{0}\right) & =d_{n}^{-1}\left\{v_{n}\left(t_{0}+s d_{n}\right)-v_{n}\left(t_{0}\right)\right\}, \\
\tilde{g}_{n}(s) & =d_{n}^{-1}\left\{x_{b, n}\left(t_{0}+s d_{n}\right)-x_{b, n}\left(t_{0}\right)\right\} .
\end{aligned}
$$

This decomposes the rescaling of $x_{n}$ as

$$
d_{n}^{-1}\left\{x_{n}\left(t_{0}+s d_{n}\right)-x_{n}\left(t_{0}\right)\right\}=\tilde{g}_{n}(s)+\tilde{w}_{n}\left(s ; t_{0}\right) .
$$

However, due to the fact that the final estimator needs to be centered at the estimand $x\left(t_{0}\right)$ and not at the preliminary estimator $x_{n}\left(t_{0}\right)$, it is more convenient to introduce the following rescaling

$$
\begin{aligned}
\tilde{v}_{n}\left(s ; t_{0}\right) & =d_{n}^{-1} v_{n}\left(t_{0}+s d_{n}\right) \\
& =\tilde{w}_{n}\left(s ; t_{0}\right)+d_{n}^{-1} v_{n}\left(t_{0}\right), \\
g_{n}(s) & =d_{n}^{-1}\left\{x_{b, n}\left(t_{0}+s d_{n}\right)-x\left(t_{0}\right)\right\} \\
& =\tilde{g}_{n}(s)+d_{n}^{-1}\left\{x_{b, n}\left(t_{0}\right)-x\left(t_{0}\right)\right\},
\end{aligned}
$$

so that

$$
y_{n}(s):=g_{n}(s)+\tilde{v}_{n}\left(s ; t_{0}\right)=d_{n}^{-1}\left\{x_{n}\left(t_{0}+s d_{n}\right)-x\left(t_{0}\right)\right\} .
$$

This definition of the rescaled deterministic and stochastic parts is slightly different from the one in Anevski and Hössjer [2], and is due to the fact that we only treat the case where the preliminary estimator and the final estimator have the same rates of convergence, in which case our definition is more convenient, whereas in Anevski and Hössjer [2] other possibilities occur.

The limit distribution results will be derived using a classical two-step procedure, cf. e.g. Prakasa Rao [32]: A local limit distribution is first obtained, under Assumption 1, stating that the estimator $T\left(x_{n}\right)$ converges weakly in a local and shrinking neighbourhood around a fixed point. Then it is shown, under Assumption 2, that the limit distribution of $T\left(x_{n}\right)$ is entirely determined by its behaviour in this shrinking neighbourhood.

Assumption 1. There exists a stochastic process $\tilde{v}\left(\cdot ; t_{0}\right) \neq 0$ such that

$$
\tilde{v}_{n}\left(\cdot ; t_{0}\right) \stackrel{\mathcal{L}}{\rightarrow} \tilde{v}\left(\cdot ; t_{0}\right)
$$


on $C(-\infty, \infty)$ as $n \rightarrow \infty$. The functions $\left\{x_{b, n}\right\}_{n \geq 1}$ are monotone and there are constants $A<0$ and $\Delta \in \mathbb{R}$ such that for each $c>0$,

$$
\sup _{|s| \leq c}\left|g_{n}(s)-(A s+\Delta)\right| \rightarrow 0
$$

as $n \rightarrow \infty$.

In the applications typically

$$
\begin{aligned}
& A=\lim _{n \rightarrow \infty} \frac{\tilde{g}_{n}(s)}{s}=x^{\prime}\left(t_{0}\right), \\
& \Delta=\lim _{n \rightarrow \infty} d_{n}^{-1}\left\{x_{b, n}\left(t_{0}\right)-x\left(t_{0}\right)\right\},
\end{aligned}
$$

so that $A$ is the local asymptotic linear term and $\Delta$ is the local asymptotic bias, both properly normalized, of the preliminary estimator $x_{n}$. Define the (limit) function

$$
y(s)=A s+\Delta+\tilde{v}\left(s ; t_{0}\right) .
$$

We next give a condition that enables a definition of the monotone rearrangement for processes. Let $\left\{z_{n}\right\}$ be an arbitrary sequence of stochastic processes.

Assumption 2. Let $I_{0}$ be a given compact interval and $\delta>0$. There exists a positive constant $c=c(\delta)$ such that $[-c, c] \supset I_{0}$ and a finite positive $M=M(\delta)$ such that

$$
\liminf _{n \rightarrow \infty} P\left\{\inf _{s \in\left(-c, \sup I_{0}\right)} z_{n}(s)>-M, \sup _{s \in(c, \infty)} z_{n}(s)<-M\right\}>1-\delta
$$

and

$$
\liminf _{n \rightarrow \infty} P\left\{\inf _{s \in(-\infty,-c)} z_{n}(s)>+M, \sup _{s \in\left(\inf I_{0}, c\right)} z_{n}(s)<+M\right\}>1-\delta .
$$

Note that in the applications typically both $c(\delta) \rightarrow \infty$ and $M(\delta) \rightarrow \infty$ as $\delta \downarrow 0$. There is no restriction in assuming this, so in the sequel we assume that $\lim _{\delta \rightarrow 0} c(\delta)=\infty$ and $\lim _{c \rightarrow \infty} \delta(c)=0$. Denote $T_{c}=T_{[-c, c]}$. Consider $D_{n}(\delta)=D_{n}(\delta(c))$ as the set of $\omega$ such that it is possible to define the monotone rearrangement $\left.T\left(z_{n}\right)\right|_{I_{0}}$ of $z_{n}$ on $I_{0}$.

Lemma 5. Let $I_{0}$ be a finite and fixed interval in $\mathbb{R}$, and $\left\{z_{n}\right\}$ be a sequence of continuous stochastic processes on $\mathbb{R}$ such that Assumption 2 holds. Then

$$
\begin{array}{r}
\lim _{c \rightarrow \infty} \liminf _{n \rightarrow \infty} P\left[D_{n}(\delta(c))\right]=1, \\
\lim _{c \rightarrow \infty} \liminf _{n \rightarrow \infty} P\left(\sup _{I_{0}}\left|T_{c}\left(z_{n}\right)(\cdot)-T\left(z_{n}\right)(\cdot)\right|=0\right)=1 .
\end{array}
$$


Proof. Let $\delta>0$ be arbitrary. Let $A_{n}(\delta, c, M)$ and $B_{n}(\delta, c, M)$ be the sets for which the probabilities are bounded in (14) and (15), respectively, for some finite $c=c(\delta)$. Then, using Theorem 3 with $I_{1}=[-c, c]$ and $I_{0}=I$, it follows that $A_{n} \cap B_{n} \subset\left\{\sup _{I}\left|T_{c}\left(z_{n}\right)-T_{J}\left(z_{n}\right)\right|=0\right.$ for each compact interval $J \supset[-c, c]\}:=C_{n}(\delta, c, M)$. Therefore $\liminf _{n \rightarrow \infty} P\left(C_{n}(\delta, c, M)\right) \geq 1-2 \delta$. Note that $C_{n}(\delta, c, M)$ is included in the set $D_{n}(\delta)$ on which it is possible to define $\left.T\left(z_{n}\right)\right|_{I_{0}}$, namely as $\left.\lim _{k \rightarrow \infty} T_{k}\left(z_{n}\right)\right|_{I_{0}}$, and which is further included in the set $E_{n}(\delta, c):=\left\{\sup _{I}\left|T_{c}\left(z_{n}\right)-T\left(z_{n}\right)\right|=\right.$ $0\}$. A priori the definition of $T$ depends on $\delta$, so that $\left.T\left(z_{n}\right)\right|_{I_{0}}=\left.T^{\delta}\left(z_{n}\right)\right|_{I_{0}}$. We will show, however, that the definition is independent of $\delta$. In fact, consider $\delta_{1}<\delta_{2}$, so that $c_{1}=c\left(\delta_{1}\right)>c_{2}=$ $c\left(\delta_{2}\right)$ and $M_{1}=M\left(\delta_{1}\right)>M_{2}=M\left(\delta_{2}\right)$. Then by the triangle inequality

$$
\sup _{I_{0}}\left|T^{\delta_{1}}\left(z_{n}\right)-T^{\delta_{2}}\left(z_{n}\right)\right| \leq \sup _{I_{0}}\left|T^{\delta_{1}}\left(z_{n}\right)-T_{c_{1}}\left(z_{n}\right)\right|+\sup _{I_{0}}\left|T_{\tilde{J}}\left(z_{n}\right)-T^{\delta_{2}}\left(z_{n}\right)\right|,
$$

with $\tilde{J}:=\left[-c_{1}, c_{1}\right]$.

Then the first term on the right-hand side is zero on $C_{n}\left(\delta_{1}, c_{1}, M_{1}\right)$, since $C_{n}\left(\delta_{1}, c_{1}, M_{1}\right) \subset$ $E_{n}\left(\delta_{1}\right)$, and the second term on the right-hand side is zero on $C_{n}\left(\delta_{2}, c_{2}, M_{2}\right)$ since $\tilde{J}$ is a compact set containing $\left[-c_{2}, c_{2}\right]$. Therefore, on the set $C_{n}\left(\delta_{1}, c_{1}, M_{1}\right) \cap C_{n}\left(\delta_{2}, c_{2}, M_{2}\right)$ the lefthand side is zero, and thus $\left.T^{\delta_{1}}\right|_{I_{0}}=\left.T^{\delta_{2}}\right|_{I_{0}}$ on that set. Note also that $P\left(D_{n}\left(\delta_{1}\right) \cap D_{n}\left(\delta_{2}\right)\right) \geq$ $P\left(C_{n}\left(\delta_{1}, c_{1}, M_{1}\right) \cap C_{n}\left(\delta_{2}, c_{2}, M_{2}\right)\right) \geq 1-2 \delta_{1}-2 \delta_{2}$. Thus, $\left.T^{\delta_{1}}\right|_{I_{0}}=\left.T^{\delta_{2}}\right|_{I_{0}}$ when both definitions exist, and they do with an as high probability as desired. This shows that the definition of $T$ does not depend on $\delta$.

Now, since $\delta>0$ is arbitrary, letting $\delta \downarrow 0$ and noting that this implies that $c \rightarrow \infty$, and using

$$
P\left(E_{n}(\delta, c)\right) \geq P\left(C_{n}(\delta, c, M)\right) \geq 1-2 \delta
$$

proves the second statement of the lemma. Noting that $D_{n} \supset C_{n}(\delta, c, M)$ proves the first statement of the lemma.

The next result is the main limit distribution result, stating that the rescaled estimator converges in distribution to a "universal" limit random variable $T(y)(0)$. The existence of the limit r.v. is made explicit in the proof of the theorem, we can for now define it (when it exists, and the proof of the following theorem shows that the limit exists as soon as $y$ satisfies Assumption 2) as a limit in probability

$$
T(y)(0) \stackrel{P}{=} \lim _{c \rightarrow \infty} T_{c}(y)(0) .
$$

In the following theorem, $J \subset \mathbb{R}$ will be a (finite or infinite) interval, in our applications $J=$ $[0,1]$ or $J=\mathbb{R}^{+}$.

Theorem 4. Let $J \subset \mathbb{R}$ be an interval, and $t_{0}$ be a fixed point belonging to the interior of $J$. Suppose Assumption 1 holds. Assume moreover that $\left\{y_{n}\right\}_{n \geq 1}$ and y are continuous processes and that Assumption 2 holds for both $\left\{y_{n}\right\}_{n \geq 1}$ and y respectively defined by (12) and (13). Then

$$
d_{n}^{-1}\left[T_{J}\left(x_{n}\right)\left(t_{0}\right)-x\left(t_{0}\right)\right] \stackrel{\mathcal{L}}{\rightarrow} T\left[A \cdot+\tilde{v}\left(\cdot ; t_{0}\right)\right](0)+\Delta,
$$

as $n \rightarrow \infty$. 
Proof. Let $c$ be a positive and finite constant and denote $T_{c, n}=T_{\left[t_{0}-c d_{n}, t_{0}+c d_{n}\right]}$. We can decompose

$$
\begin{aligned}
d_{n}^{-1}\left\{T_{J}\left(x_{n}\right)\left(t_{0}\right)-x\left(t_{0}\right)\right\}= & d_{n}^{-1}\left\{T_{J}\left(x_{n}\right)\left(t_{0}\right)-T_{c, n}\left(x_{n}\right)\left(t_{0}\right)\right\} \\
& +d_{n}^{-1}\left\{T_{c, n}\left(x_{n}\right)\left(t_{0}\right)-x\left(t_{0}\right)\right\}
\end{aligned}
$$

Let us first consider the second term of the right-hand side of (17) and introduce

$$
\chi_{n}(s):=x_{n}\left(t_{0}+s d_{n}\right)=x\left(t_{0}\right)+d_{n} y_{n}(s) .
$$

Applying Lemma 3 leads to

$$
T_{c, n}\left(x_{n}\right)\left(t_{0}+s d_{n}\right)=T_{c}\left(\chi_{n}\right)(s)=d_{n} T_{c}\left(y_{n}\right)(s)+x\left(t_{0}\right),
$$

which gives

$$
d_{n}^{-1}\left\{T_{c, n}\left(x_{n}\right)\left(t_{0}\right)-x\left(t_{0}\right)\right\}=T_{c}\left(y_{n}\right)(0)
$$

Assumption 1 implies that $y_{n} \stackrel{\mathcal{L}}{\rightarrow} y$ on $C[-c, c]$, with $y$ defined in (13). Applying the continuous mapping theorem on $T_{c}$, cf. Theorem 1 , proves

$$
d_{n}^{-1}\left\{T_{c, n}\left(x_{n}\right)\left(t_{0}\right)-x\left(t_{0}\right)\right\} \stackrel{\mathcal{L}}{\rightarrow} T_{c}(y)(0)
$$

as $n \rightarrow \infty$. Lemma 5 via Assumption 2 with $z_{n}=y$ shows that we can define the limit random variable $T(y)(0)$ as a limit in probability so that, as $c \rightarrow \infty$,

$$
T_{c}(y)(0) \stackrel{P}{\rightarrow} T(y)(0)
$$

Next, we consider the first term of the right-hand side of (17). Let $\nabla$ be a positive and finite constant and denote $A_{n, \nabla}=\left[t_{0}-\nabla d_{n}, t_{0}+\nabla d_{n}\right]$. From (18) and Lemma 3 it follows that

$$
\sup _{A_{n, \nabla}} d_{n}^{-1}\left|T_{c, n}\left(x_{n}\right)(\cdot)-T_{J}\left(x_{n}\right)(\cdot)\right|=\sup _{[-\nabla, \nabla]}\left|T_{c}\left(y_{n}\right)(\cdot)-T_{J_{n, t_{0}}}\left(y_{n}\right)(\cdot)\right|,
$$

with $y_{n}$ as defined in (12). Using Lemma 5 with $I=[-\nabla, \nabla]$ shows that

$$
d_{n}^{-1}\left\{T_{c, n}\left(x_{n}\right)\left(t_{0}\right)-T_{J}\left(x_{n}\right)\left(t_{0}\right)\right\} \stackrel{P}{\rightarrow} 0
$$

when $n \rightarrow \infty$.

Let first $n$ tend to infinity in (17), and apply Slutsky's theorem with the use of (19), (21). Note that when $c \rightarrow \infty$, (20) gives the result.

Remark 4. The approach for deriving the limit distributions is similar to the general approach in Anevski and Hössjer [2] with a preliminary estimator that is made monotone via the $\mathbb{L}^{2}$ projection on the space of monotone functions. There are however a few differences: 
- Anevski and Hössjer look at rescaling of an integrated preliminary estimator of the monotone functions, whereas we rescale the estimator directly. Our approach puts a stronger assumption on the asymptotic properties of the preliminary estimator, which is however traded off against weaker conditions on the map $T$, since we only have to assume that the map $T$ is continuous; had we dealt with rescaling as in Anevski and Hössjer we would have had to prove that the composition $\frac{d}{d t}(\tilde{T})$ (with $\tilde{T}$ defined by $\tilde{T}(F)(t)=\int_{0}^{t} T\left(F^{\prime}\right)(u) d u$ ) is a continuous map, which is generally not true for $T$ equal to the monotone rearrangement map; it is however true, under certain conditions, for $\tilde{T}$ equal to the least concave minorant map (when $T$ becomes the $\mathbb{L}^{2}$-projection on the space of monotone functions), cf. Proposition 2 in Anevski and Hössjer [2].

- We are able to do rescaling for the preliminary estimator directly since it is a smooth function. On the contrary, for some of the cases treated in Anevski and Hössjer this is not possible, for example, for the isotonic regression and the NPMLE of a monotone density the rescaled stochastic part is asymptotically white noise. As a consequence our rescaled deterministic function is assumed to be approximated by a linear function, whereas the rescaled deterministic function in Anevski and Hössjer [2] is assumed to be approximated by a convex or concave function.

- The rescaling is here centered at $x\left(t_{0}\right)$, and not at $x_{n}\left(t_{0}\right)$, which makes it more convenient to apply the limit distribution result we get.

The rest of this section is to apply the previous result to two nonparametric inference problems: next subsection deals with the estimation of a monotone density function, and the last one with estimating a monotone regression function. Limit distributions for estimators of a marginal decreasing density $f$ for stationary weakly dependent data with marginal density $f$ as well as of a monotone regression function $m$ with stationary errors, that are weakly or strongly dependent, will be derived.

All limit distribution results stated will be for processes in $C(-\infty, \infty)$ with the uniform metric on compact intervals and the Borel $\sigma$-algebra.

\subsection{Application to monotone density estimation}

For the density estimation problem, let $\left\{t_{i}\right\}_{i=1}^{\infty}$ denote a stationary process with marginal density function $f$. Define the empirical distribution function $F_{n}(t)=\frac{1}{n} \sum_{i=1}^{n} 1_{\left\{t_{i} \leq t\right\}}$ and the centered empirical process $F_{n}^{0}(t)=\frac{1}{n} \sum_{i=1}^{n}\left(1_{\left\{t_{i} \leq t\right\}}-F(t)\right)$. Consider a sequence $\delta_{n}$ such that $\delta_{n} \downarrow 0, n \delta_{n} \uparrow \infty$ as $n \rightarrow \infty$, and define the centered empirical process locally around $t_{0}$ on scale $\delta_{n}$ as

$$
\begin{aligned}
w_{n, \delta_{n}}\left(s ; t_{0}\right)= & \sigma_{n, \delta_{n}}^{-1} n\left\{F_{n}^{0}\left(t_{0}+s \delta_{n}\right)-F_{n}^{0}\left(t_{0}\right)\right\} \\
= & \sigma_{n, \delta_{n}}^{-1} \sum_{i=1}^{n}\left(1_{\left\{t_{i} \leq t_{0}+s \delta_{n}\right\}}-1_{\left\{t_{i} \leq t_{0}\right\}}\right. \\
& \left.-F\left(t_{0}+s \delta_{n}\right)+F\left(t_{0}\right)\right),
\end{aligned}
$$


where

$$
\begin{aligned}
\sigma_{n, \delta_{n}}^{2} & =\operatorname{Var}\left[n\left\{F_{n}^{0}\left(t_{0}+\delta_{n}\right)-F_{n}^{0}\left(t_{0}\right)\right\}\right] \\
& =\operatorname{Var}\left[\sum_{i=1}^{n}\left\{1_{\left\{t_{0}<t_{i} \leq t_{0}+\delta_{n}\right\}}-F\left(t_{0}+\delta_{n}\right)+F\left(t_{0}\right)\right\}\right] .
\end{aligned}
$$

In this section, we introduce a (monotone) estimator of a monotone density function for stationary data, for which we derive consistency and limit distributions.

Let $t_{1}, t_{2}, \ldots$ denote a stationary process with marginal density function $f$ lying in the class of decreasing density functions on $\mathbb{R}^{+}$, and define the following estimator of the marginal decreasing density for the sequence $\left\{t_{i}\right\}_{i \geq 1}$ : Let $x_{n}(t)=(n h)^{-1} \sum_{i=1}^{n} k\left\{\left(t-t_{i}\right) / h\right\}$ be the kernel estimator of the density $f$, with $k$ a bounded density function supported on $[-1,1]$ such that $\int k^{\prime}(u) d u=0$, and $h>0$ the bandwidth (cf. e.g., Wand and Jones [43]), and define the (monotone) density estimate

$$
\hat{f}_{n}(t)=T\left(x_{n}\right)(t)
$$

where $T$ is the monotone rearrangement map on $\mathbb{R}^{+}$as defined in (2). Note that $\hat{f}_{n}$ is monotone and positive, and integrates to one, cf. equation (4) of Section 3.3 in Lieb and Loss [24].

A straightforward consequence of Theorem 2 and standard convergence results for the kernel density estimate is the following consistency result.

Proposition 1. The random function $\hat{f}_{n}$ defined by (22) is a uniformly consistent estimator of $f$ in probability uniformly on compact sets, and in probability in $\mathbb{L}^{p}$-norm.

In the following, the limit distributions for $\hat{f}_{n}$ in the independent and weakly dependent cases are derived. We will in particular make use of recent results on the weak convergence $w_{n, \delta_{n}} \stackrel{\mathcal{L}}{\rightarrow} w$, on $D(-\infty, \infty)$, as $n \rightarrow \infty$, for independent and weakly dependent data $\left\{t_{i}\right\}$, derived in Anevski and Hössjer [2].

The kernel estimator can be written $x_{n}=x_{b, n}+v_{n}$ with

$$
\begin{gathered}
x_{n}(t)=h^{-1} \int k^{\prime}(u) F_{n}(t-h u) d u, \\
x_{b, n}(t)=h^{-1} \int k^{\prime}(u) F(t-h u) d u, \\
v_{n}(t)=h^{-1} \int k^{\prime}(u) F_{n}^{0}(t-h u) d u .
\end{gathered}
$$

Rescaling is done on a scale $d_{n}$ that is of the same asymptotic order as $h$, so that we put $d_{n}=h$. The rescaled process is

$$
\tilde{v}_{n}\left(s ; t_{0}\right)=c_{n} \int k^{\prime}(u) w_{n, d_{n}}\left(s-u ; t_{0}\right) d u,
$$

with $c_{n}=d_{n}^{-1}(n h)^{-1} \sigma_{n, d_{n}}$. 
Theorem 5. Let $\left\{t_{i}\right\}_{i \geq 1}$ be a stationary sequence with a monotone marginal density function $f$ such that $\sup _{t \in I_{t_{0}}} f^{\prime}(t)<0$ and $f \in C^{1}\left(I_{t_{0}}\right)$ for an open interval $I_{t_{0}} \ni t_{0}$ where $t_{0}>0$. Assume that $\mathbb{E} t_{i}^{4}<\infty$. Let $x_{n}$ be the kernel density function defined above, with $k$ a bounded and compactly supported density such that $k^{\prime}$ is bounded. Suppose that one of the following conditions holds:

(a) $\left\{t_{i}\right\}_{i \geq 1}$ is an i.i.d. sequence,

(b) (1) $\left\{t_{i}\right\}_{i \geq 1}$ is a stationary $\phi$-mixing sequence with $\sum_{i=1}^{\infty} \phi^{1 / 2}(i)<\infty$;

(2) $f\left(t_{0}\right)=F^{\prime}\left(t_{0}\right)$ exists, as well as the joint density $f_{k}\left(s_{1}, s_{2}\right)$ of $\left(t_{1}, t_{1+k}\right)$ on $\left[t_{0}-\right.$ $\left.\delta, t_{0}+\delta\right]^{2}$ for some $\delta>0$, and $k \geq 1$;

(3) $\sum_{k=1}^{\infty} M_{k}<\infty$ holds, for $M_{k}=\sup _{t_{0}-\delta \leq s_{1}, s_{2} \leq t_{0}+\delta}\left|f_{k}\left(s_{1}, s_{2}\right)-f\left(s_{1}\right) f\left(s_{2}\right)\right|$.

Then choosing $h=a n^{-1 / 3}$ and $a>0$ an arbitrary constant, we obtain

$$
n^{1 / 3}\left\{\hat{f}_{n}\left(t_{0}\right)-f\left(t_{0}\right)\right\} \stackrel{\mathcal{L}}{\rightarrow} a T\left[f^{\prime}\left(t_{0}\right) \cdot+\tilde{v}\left(\cdot ; t_{0}\right)\right](0)+f^{\prime}\left(t_{0}\right) a \int u k(u) d u,
$$

as $n \rightarrow \infty$, where $\tilde{v}(s ; t)$ is as in $(42)$, with $c=a^{-3 / 2} f\left(t_{0}\right)^{1 / 2}$, and $w$ a standard two sided Brownian motion.

Proof. If $k^{\prime}$ is bounded and $k$ has compact support, the continuity of the map

$$
C(-\infty, \infty) \ni z(s) \quad \mapsto \quad \int z(s-u) k^{\prime}(u) d u \in C(-\infty, \infty)
$$

implies that, choosing $d_{n}$ such that $c_{n} \rightarrow c$ as $n \rightarrow \infty$ for some constant $c$, one gets:

$$
\tilde{v}_{n}\left(s ; t_{0}\right) \stackrel{\mathcal{L}}{\rightarrow} c \int k^{\prime}(u) w\left(s-u ; t_{0}\right) d u=: \tilde{v}\left(s ; t_{0}\right),
$$

on $C(-\infty, \infty)$, as $n \rightarrow \infty$, thanks to the continuous mapping theorem. Here $w$ is the weak limit of $\left\{w_{n}\right\}$. Theorems 7 and 8 of Anevski and Hössjer [2] state that $w_{n, \delta_{n}}\left(s, t_{0}\right) \stackrel{\mathcal{L}}{\rightarrow} B(s)$ on $D(-\infty, \infty)$ under the respective assumptions in (a) and (b), where $B(s)$ is a two sided standard Brownian motion. This establishes the first part of Assumption 1 for both cases (a) and (b). Next notice that $x_{b, n}(t)=h^{-1} \int k\left(\frac{t-u}{h}\right) f(u) d u$ is monotone. A change of variable and a Taylor expansion in $x_{b, n}$ prove the second part of Assumption 1 with $A=f^{\prime}\left(t_{0}\right)$ and

$$
d_{n}^{-1}\left\{x_{b, n}\left(t_{0}\right)-f\left(t_{0}\right)\right\} \rightarrow f^{\prime}\left(t_{0}\right) \int u k(u) d u=\Delta .
$$

The statement of Assumption 2 is relegated to the appendix, see Corollary 2 in the supplemental article (Anevski and Fougères [1]). Theorem 5 therefore holds as an application of Theorem 4.

Let us finally check that the scale $d_{n}$ can be chosen so that $c_{n} \rightarrow c$, as assumed at the beginning of the proof: 
- Independent data case (a): We have $\sigma_{n, d_{n}}^{2} \sim n d_{n} f\left(t_{0}\right)$, so that

$$
d_{n}^{-1}(n h)^{-1} \sigma_{n, d_{n}} \sim d_{n}^{-3 / 2} n^{-1 / 2} f\left(t_{0}\right)^{1 / 2} .
$$

Choosing $d_{n}=a n^{-1 / 3}$ we get $c=a^{-3 / 2} f\left(t_{0}\right)^{1 / 2}$.

- Mixing data case (b): Similar to the proof of case (a).

Remark 5. The present estimator was first proposed for independent data by Fougères [16], who stated the strong consistency uniformly over $\mathbb{R}^{+}$for $T\left(f_{n}\right)$ and derived some partial results for the limit distribution. The results for the monotone density function estimator are similar to the results for the Grenander estimator (the NPMLE) of a monotone density, in that we have cube root asymptotics and a limit random variable that is a nonlinear functional of a Gaussian process, for independent and weak dependent data; see Prakasa Rao [32] and Wright [44] for the independent data cases, and Anevski and Hössjer [2] for the weak dependent data cases. In our case however we obtain one extra term that arises from the bias in the kernel estimator. Our estimator is really closer in spirit to the estimator obtained by projecting the kernel estimator on the space of monotone functions (i.e. kernel estimation followed by isotonic regression) first proposed by Anevski and Hössjer [2]; note that we obtain the same bias term as in Anevski and Hössjer [2].

Remark 6. The results for the long range dependence case is similar to the result for the isotonic regression of a kernel estimator, cf. Anevski and Hössjer [2]. In this situation, $\tilde{v}_{n}\left(s ; t_{0}\right)$ is asymptotically a linear function of $s$ with a random slope, implying that the monotone rearrangement of $g_{n}+\tilde{v}_{n}$ is just $g_{n}+\tilde{v}_{n}$ which evaluated at zero is zero. This is due to the fact that for long range dependent data the limit process of the empirical process is a deterministic function multiplied by a random variable, cf. the remark after Theorem 12 in Anevski and Hössjer [2]. Thus, the limit distribution for the final estimator for long range dependent data is the same as the limit distribution for the kernel estimator itself, that is, $n^{d / 2}\left\{\hat{f}_{n}(t)-f(t)\right\}$ and $n^{d / 2}\left\{f_{n}(t)-f(t)\right\}$ have the same distributional limit. See Csörgö and Mielniczuk [12] for a derivation of this limit distribution.

\subsection{Application to monotone regression function estimation}

For the regression function estimation problem, let $\left\{\varepsilon_{i}\right\}_{i=-\infty}^{\infty}$ be a stationary sequence of random variables with $E\left(\varepsilon_{i}\right)=0$ and $\operatorname{Var}\left(\varepsilon_{i}\right)=\sigma^{2}<\infty$. Let $\sigma_{n}^{2}=\operatorname{Var}\left(\sum_{i=1}^{n} \varepsilon_{i}\right)$. The two sided partial sum process $w_{n}$ is defined by

$$
w_{n}\left(t_{i}+\frac{1}{2 n}\right)= \begin{cases}\frac{1}{\sigma_{n}}\left(\frac{\varepsilon_{0}}{2}+\sum_{j=1}^{i} \varepsilon_{j}\right), & i=0,1,2, \ldots, \\ \frac{1}{\sigma_{n}}\left(-\frac{\varepsilon_{0}}{2}-\sum_{j=i+1}^{-1} \varepsilon_{j}\right), & i=-1,-2, \ldots,\end{cases}
$$

and linearly interpolated between these points. Note that $w_{n} \in C(\mathbb{R})$. 
Let $\operatorname{Cov}(k)=E\left(\xi_{1} \xi_{1+k}\right)$ denote the covariance function of a generic stationary sequence $\left\{\xi_{i}\right\}$, and distinguish between three cases (of which (a) is a special case of (b)).

(a) Independence: the $\varepsilon_{i}$ are independent.

(b) Weak dependence: $\sum_{k}|\operatorname{Cov}(k)|<\infty$.

(c) Strong (long range) dependence: $\sum_{k}|\operatorname{Cov}(k)|=\infty$.

Weak dependence can be further formalized using mixing conditions as follows: Define two $\sigma$-algebras of a sequence $\left\{\xi_{i}\right\}$ as $\mathcal{F}_{k}=\sigma\left\{\xi_{i}: i \leq k\right\}$ and $\overline{\mathcal{F}}_{k}=\sigma\left\{\xi_{i}: i \geq k\right\}$, where $\sigma\left\{\xi_{i}: i \in I\right\}$ denotes the $\sigma$-algebra generated by $\left\{\xi_{i}: i \in I\right\}$. The stationary sequence $\left\{\xi_{i}\right\}$ is said to be " $\varphi$ mixing" or " $\alpha$-mixing" respectively if there is a function $\varphi(n)$ or $\alpha(n) \rightarrow 0$ as $n \rightarrow \infty$, such that

$$
\begin{array}{r}
\sup _{A \in \overline{\mathcal{F}}_{n}}\left|P\left(A \mid \mathcal{F}_{0}\right)-P(A)\right| \leq \varphi(n), \\
\sup _{A \in \mathcal{F}_{0}, B \in \overline{\mathcal{F}}_{n}}|P(A B)-P(A) P(B)| \leq \alpha(n),
\end{array}
$$

respectively. Finally, long range dependence is usually formalized using subordination or assuming the processes are linear; we will treat only (Gaussian) subordination.

In this section, we introduce an estimator of a monotone regression function. We derive consistency and limit distributions, under general dependence assumptions.

Assume $m$ is a $C^{1}$-function on a compact interval $J \subset \mathbb{R}$, say $J=[0,1]$ for simplicity; let $\left(y_{i}, t_{i}\right), i=1, \ldots, n$ be pairs of data satisfying

$$
y_{i}=m\left(t_{i}\right)+\varepsilon_{i}
$$

where $t_{i}=i / n$. Define $\bar{y}_{n}:[1 / n, 1] \mapsto \mathbb{R}$ by linear interpolation of the points $\left\{\left(t_{i}, y_{i}\right)\right\}_{i=1}^{n}$, and let

$$
x_{n}(t)=h^{-1} \int k((t-u) / h) \bar{y}_{n}(u) d u
$$

be the Gasser-Müller kernel estimate of $m(t)$, cf. Gasser and Müller [17], where $k$ is a density in $\mathbf{L}^{2}(\mathbb{R})$ with compact support, for simplicity take $\operatorname{supp}(k)=[-1,1]$. Let $h$ be the bandwidth, for which we assume that $h \rightarrow 0, n h \rightarrow \infty$.

To define a monotone estimator of $m$, we put

$$
\tilde{m}(t)=T_{[0,1]}\left(x_{n}\right)(t), \quad t \in J,
$$

where $T_{[0,1]}$ is the monotone rearrangement map on [0,1]. A straightforward application of Theorem 2 and standard consistency results for regression function estimators imply the following consistency result.

Proposition 2. The random function $\tilde{m}$ defined by (27) is a uniformly consistent estimator of $m$ in probability uniformly on compact sets, and in probability in $\mathbb{L}^{p}$ norm. 
Clearly $x_{n}(t)=x_{b, n}(t)+v_{n}(t)$, with

$$
\begin{gathered}
x_{b, n}(t)=h^{-1} \int k\left(\frac{t-u}{h}\right) \bar{m}_{n}(u) d u, \\
v_{n}(t)=h^{-1} \int k\left(\frac{t-u}{h}\right) \bar{\varepsilon}_{n}(u) d u,
\end{gathered}
$$

where the functions $\bar{m}_{n}$ and $\bar{\varepsilon}_{n}$ are obtained by linear interpolation of $\left\{\left(t_{i}, m\left(t_{i}\right)\right)\right\}_{i=1}^{n}$ and $\left\{\left(t_{i}, \varepsilon_{i}\right)\right\}_{i=1}^{n}$ respectively. For the deterministic term $x_{b, n}(t) \rightarrow x_{b}(t)=m(t)$, as $n \rightarrow \infty$. Note that $\bar{m}_{n}$, and thus also $x_{b, n}$, is monotone. Put

$$
\bar{w}_{n}(t)=\frac{n}{\sigma_{n}} \int_{0}^{t} \bar{\varepsilon}_{n}(u) d u .
$$

Since $\operatorname{supp}(k)=[-1,1]$ and if $t \in(1 / n+h, 1-h)$, from a partial integration and change of variable, we obtain

$$
v_{n}(t)=\frac{\sigma_{n}}{n h} \int k^{\prime}(u) \bar{w}_{n}(t-u h) d u
$$

It can be shown that $\bar{w}_{n}$ and $w_{n}$ are asymptotically equivalent for all dependence structures treated in this paper. Let us now recall how the two sided partial sum process behaves in the different cases of dependence we consider:

(a) When the $\varepsilon_{i}$ are independent, we have the classical Donsker theorem, cf. Billingsley [4], implying that

$$
w_{n} \stackrel{\mathcal{L}}{\rightarrow} B
$$

as $n \rightarrow \infty$, with $B$ a two sided standard Brownian motion on $C(\mathbb{R})$.

(b) Define

$$
\kappa^{2}=\operatorname{Cov}(0)+2 \sum_{k=1}^{\infty} \operatorname{Cov}(k)
$$

Assumption 3 ( $\phi$-mixing). Assume $\left\{\varepsilon_{i}\right\}_{i \in \mathbf{Z}}$ is a stationary $\phi$-mixing sequence with $E \varepsilon_{i}=0$ and $E \varepsilon_{i}^{2}<\infty$. Assume further $\sum_{k=1}^{\infty} \phi(k)^{1 / 2}<\infty$ and $\kappa^{2}>0$ in (30).

Assumption 4 ( $\boldsymbol{\alpha}$-mixing). Assume $\left\{\varepsilon_{i}\right\}_{i \in \mathbf{Z}}$ is a stationary $\alpha$-mixing sequence with $E \varepsilon_{i}=0$ and $E \varepsilon_{i}^{4}<\infty, \kappa^{2}>0$ in (30) and $\sum_{k=1}^{\infty} \alpha(k)^{1 / 2-\varepsilon}<\infty$, for some $\varepsilon>0$.

Assumption 3 or 4 imply that $\sigma_{n}^{2} \rightarrow \kappa^{2}$ and that Donsker's result (29) is valid, cf. Anevski and Hössjer [2] and references therein. 
(c) We model long range dependent data $\left\{\varepsilon_{i}\right\}_{i \geq 1}$ using Gaussian subordination: More precisely, we write $\varepsilon_{i}=g\left(\xi_{i}\right)$ with $\left\{\xi_{i}\right\}_{i \in \mathbf{Z}}$ a stationary Gaussian process with mean zero and covariance function $\operatorname{Cov}(k)=E\left(\xi_{i} \xi_{i+k}\right)$ such that $\operatorname{Cov}(0)=1$ and $\operatorname{Cov}(k)=k^{-d} l_{0}(k)$, with $l_{0}$ a slowly varying function at infinity ${ }^{1}$ and $0<d<1$ fixed. Furthermore $g: \mathbb{R} \mapsto \mathbb{R}$ is a measurable function with $E\left\{g\left(\xi_{1}\right)^{2}\right\}<\infty$. An expansion $g\left(\xi_{i}\right)$ in Hermite polynomials is available

$$
g\left(\xi_{i}\right)=\sum_{k=r}^{\infty} \frac{1}{k !} \eta_{k} h_{k}\left(\xi_{i}\right)
$$

where equality holds as a limit in $L^{2}(\varphi)$, with $\varphi$ the standard Gaussian density function. The functions $h_{k}(t)=t^{-k}(d / d t)^{k}\left(t^{k} e^{-t^{2}}\right)$ are the Hermite polynomials of order $k$, the functions

$$
\eta_{k}=E\left\{g\left(\xi_{1}\right) h_{k}\left(\xi_{1}\right)\right\}=\int g(u) h_{k}(u) \phi(u) d u,
$$

are the $L^{2}(\varphi)$-projections on $h_{k}$, and $r$ is the index of the first non-zero coefficient in the expansion. Assuming that $0<d r<1$, the subordinated sequence $\left\{\varepsilon_{i}\right\}_{i \geq 1}$ exhibits long range dependence (see, e.g., Taqqu [35,36]), and Taqqu [35] also shows that

$$
\sigma_{n}^{-1} \sum_{i \leq n t} g\left(\xi_{i}\right) \stackrel{\mathcal{L}}{\rightarrow} z_{r, \beta}(t),
$$

in $D[0,1]$ equipped with the Skorokhod topology, with variance $\sigma_{n}^{2}=\operatorname{Var}\left\{\sum_{i=1}^{n} g\left(\xi_{i}\right)\right\}=$ $\eta_{r}^{2} n^{2-r d} l_{1}(n)(1+o(1))$, where

$$
l_{1}(k)=\frac{2}{r !(1-r d)(2-r d)} l_{0}(k)^{r} .
$$

The limit process $z_{r, \beta}$ is in $C[0,1]$ a.s., and is self similar with parameter

$$
\beta=1-r d / 2 .
$$

The process $z_{1, \beta}(t)$ is fractional Brownian motion, $z_{2, \beta}(t)$ is the Rosenblatt process, and the processes $z_{r, \beta}(t)$ are all non-Gaussian for $r \geq 2$, cf. Taqqu [35]. From these results follows a two sided version of Taqqu's result stating the behavior of the two sided partial sum process:

$$
w_{n} \stackrel{\mathcal{L}}{\rightarrow} B_{r, \beta},
$$

in $D(-\infty, \infty)$, as $n \rightarrow \infty$, where $B_{r, \beta}$ are the two sided versions of the processes $z_{r, \beta}$.

${ }^{1}$ i.e. $l_{0}(t k) / l_{0}(t) \rightarrow 1$ as $t \rightarrow \infty$ for each positive $k$. 
In the sequel, rescaling is done at the bandwidth rate, so that $d_{n}=h$. For $s>0$, let consider the following rescaled process:

$$
\begin{aligned}
\tilde{v}_{n}(s ; t) & =d_{n}^{-1}(n h)^{-1} \sigma_{\hat{n}} \int \bar{w}_{\hat{n}}\left(h^{-1} t+s-u\right) k^{\prime}(u) d u \\
& \underline{\mathcal{L}} d_{n}^{-1}(n h)^{-1} \sigma_{\hat{n}} \int \bar{w}_{\hat{n}}(s-u) k^{\prime}(u) d u
\end{aligned}
$$

with $\hat{n}=[n h]$ the integer part of $n h$, where the last equality holds due to the stationarity (exactly only for $t=t_{i}$ and asymptotically otherwise). Note that the right-hand side holds also for $s<0$.

With the bandwidth choice $d_{n}=h$ we obtain a non-trivial limit process $\tilde{v}$; choosing $d_{n}$ such that $d_{n} / h \rightarrow 0$ leads to a limit "process" equal to a random variable and $d_{n} / h \rightarrow \infty$ to white noise. In the first case, the limit distribution of $T\left(x_{n}\right)$ on the scale $d_{n}$ will be the constant 0 , while in the second case it will (formally) be $T\left(m^{\prime}\left(t_{0}\right) \cdot+\tilde{v}(\cdot)\right)(0)$ which is not defined ( $T$ can not be defined for generalized functions, in the sense of L. Schwartz [34]).

Theorem 6. Assume $m$ is monotone on $[0,1]$ and for some open interval $I_{t_{0}} \ni t_{0}, m \in C^{1}\left(I_{t_{0}}\right)$ and $\sup _{t \in I_{t_{0}}} m^{\prime}(t)<0$ with $t_{0} \in(0,1)$. Let $x_{n}$ be the kernel estimate of $m$ defined in (26), with a nonnegative and compactly supported kernel $k$ such that $k^{\prime}$ is bounded, and with bandwidth $h$ specified below. Suppose that one of the following conditions holds.

(a) $\left\{\varepsilon_{i}\right\}$ are independent and identically distributed, $E \varepsilon_{i}=0 ; \sigma^{2}=\operatorname{Var}\left(\varepsilon_{i}\right)<\infty$, and $h=$ $a n^{-1 / 3}$, for an arbitrary $a>0$,

(b) Assumption 3 or 4 holds, $\sigma_{n}^{2}=\operatorname{Var}\left(\sum_{i=1}^{n} \varepsilon_{i}\right), \kappa^{2}$ is defined in (30), and $h=a n^{-1 / 3}$, with $a>0$ an arbitrary constant,

(c) $\varepsilon_{i}=g\left(\xi_{i}\right)$ is a long range dependent subordinated Gaussian sequence with parameters $d$ and $r, h=l_{2}(n ; a) n^{-r d /(2+r d)}$ with $a>0$ and $n \mapsto l_{2}(n ; a)$ is a slowly varying function defined in the proof below.

Then, correspondingly, we obtain

$$
h^{-1}\left\{\tilde{m}\left(t_{0}\right)-m\left(t_{0}\right)\right\} \stackrel{\mathcal{L}}{\rightarrow} T\left[m^{\prime}\left(t_{0}\right) \cdot+\tilde{v}\left(\cdot ; t_{0}\right)\right](0)+m^{\prime}\left(t_{0}\right) \int u k(u) d u,
$$

as $n \rightarrow \infty$, where $\tilde{m}$ is defined in (27),

$$
\tilde{v}(s ; t)=c \int w(s-u) k^{\prime}(u) d u
$$

and respectively,

(a) $w=B ; c=\sigma a^{-3 / 2}$,

(b) $w=B ; c=\kappa a^{-3 / 2}$,

(c) $w=B_{r, \beta} ; c=\left|\eta_{r}\right| a$ (where $\beta$ defined in (32)). 
Proof. The conclusions of the theorem follow by an application of Theorem 4 in the context of monotone regression function. Assume first that $d_{n}=h$ is such that

$$
d_{n}^{-1}(n h)^{-1} \sigma_{\hat{n}}=d_{n}^{-2} n^{-1} \sigma_{\hat{n}} \rightarrow c>0 .
$$

Then $w_{n} \stackrel{\mathcal{L}}{\rightarrow} w$ in $D(-\infty, \infty)$, using the supnorm over compact intervals metric, under the respective assumptions in (a), (b) and (c). Besides, note that if $k^{\prime}$ is bounded and $k$ has compact support, the map

$$
C(-\infty, \infty) \ni z(s) \quad \mapsto \quad \int z(s-u) k^{\prime}(u) d u \in C(-\infty, \infty)
$$

is continuous, in the supnorm over compact intervals metric. Thus, under the assumptions that $k^{\prime}$ is bounded and $k$ has compact support, the continuous mapping theorem implies that

$$
\tilde{v}_{n}(s ; t) \stackrel{\mathcal{L}}{\rightarrow} \tilde{v}(s ; t),
$$

where $\tilde{v}(s ; t)$ is defined in (43). This yields the first part of Assumption 1. Furthermore

$$
\begin{aligned}
\tilde{g}_{n}(s) & =h^{-1} \int \ell(u) \bar{m}_{n}\left(t_{0}-h u\right) d u \\
& =h^{-1} \int \ell(u) m\left(t_{0}-h u\right) d u+r_{n}(s),
\end{aligned}
$$

with $\ell(v)=k(v+s)-k(v)$ and $r_{n}$ a remainder term. Since

$$
\int v^{\lambda} \ell(v) d v= \begin{cases}0, & \text { if } \lambda=0, \\ -s, & \text { if } \lambda=1,\end{cases}
$$

it follows by a Taylor expansion of $m$ around $t_{0}$ that the first term converges towards $A s$, with $A=m^{\prime}\left(t_{0}\right)$. The remainder term is bounded for any $c>0$ as

$$
\begin{aligned}
\sup _{|s| \leq c}\left|r_{n}(s)\right| & \leq h^{-1} \sup _{|s| \leq c} \int|\ell(u)| d u \sup _{\left|u-t_{0}\right| \leq(c+1) h}\left|\bar{m}_{n}(u)-m(u)\right| \\
& =O\left(n^{-1} h^{-1}\right)=o(1) .
\end{aligned}
$$

Furthermore

$$
d_{n}^{-1}\left\{x_{b, n}\left(t_{0}\right)-m\left(t_{0}\right)\right\} \rightarrow m^{\prime}\left(t_{0}\right) \int u k(u) d u=: \Delta,
$$

as $n \rightarrow \infty$, which proves Assumption 1 .

Proof that Assumption 2 holds is relegated to the appendix, see Corollary 1 in the supplemental article (Anevski and Fougères [1]). An application of Theorem 4 then finishes the proof of Theorem 6. It only remains to check whether $d_{n}^{-1}(n h)^{-1} \sigma_{\hat{n}} \rightarrow c>0$ for the three types of dependence. 
- Independent case (a): We have $\sigma_{\hat{n}}^{2}=\sigma^{2} n d_{n}$. Thus $d_{n}^{-1}(n h)^{-1} \sigma_{\hat{n}}=\sigma n^{-1 / 2} h^{-3 / 2}$, and (45) is satisfied with $c=\sigma a^{-3 / 2}$ if $d_{n}=h=a n^{-1 / 3}$.

- Mixing case (b): The proof is similar to the proof of (a), replacing $\sigma$ by $\kappa$.

- Long range data case (c): Since $\sigma_{\hat{n}}^{2}=\eta_{r}^{2}\left(n d_{n}\right)^{2-r d} l_{1}\left(n d_{n}\right)$, if we choose $d_{n}=h$ we will have

$$
d_{n}^{-2} n^{-1} \sigma_{\hat{n}}=d_{n}^{-2} n^{-1}\left|\eta_{r}\right|\left(n d_{n}\right)^{1-r d / 2} l_{1}\left(n d_{n}\right)^{1 / 2} \rightarrow\left|\eta_{r}\right| a
$$

if and only if

$$
d_{n}=n^{-r d /(2+r d)} l_{2}(n ; a),
$$

where $l_{2}$ is another function slowly varying at infinity, implicitly defined in (37). Thus (45) follows with $c=\left|\eta_{r}\right| a$ and $h=d_{n}$ given in (47).

Remark 7. The present estimator is similar to the estimator first presented by Mammen [25]: Mammen proposed to do isotonic regression of a kernel estimator of a regression function (using bandwidth $h=n^{-1 / 5}$ ), whereas we do monotone rearrangement of a kernel estimator. Mammen's estimator was extended to dependent data and other bandwidth choices by Anevski and Hössjer [2] who derived limit distributions for weak dependent and long range dependent data that are analogous to our results; for the independent data case and bandwidth choice $h=n^{-1 / 3}$ the limit distributions are similar with rate of convergence $n^{1 / 3}$ and nonlinear maps of Gaussian processes.

\section{Limit results for density and regression function estimators with $q$ vanishing derivatives}

The results we have established in the previous section can in fact be extended to the case when the estimand $x$ is monotone and has $q$ vanishing moments at the point of interest $t_{0}$, so when $x\left(t_{0}\right) \neq 0, x^{(i)}\left(t_{0}\right)=0$ for $j=1, \ldots, q$ and $x^{(q+1)}\left(t_{0}\right)<0$. We present these results here. We will make an analogous derivation to the case when $x^{\prime}\left(t_{0}\right)<0$, and we will highlight when there is a difference to the case already treated.

We give proofs for the independent data, the weak dependent and the long range dependent cases. Our results give other rates of convergence and other (new) limit random variables. For instance, the limit results that we obtain for the independent data case will be with the (slower rate of convergence) $n^{-1 /(q+3)}$, and the limit r.v. will be of the form

$$
T(A(s)+\tilde{v}(s))(0)
$$

where $A$ is (when $k$ is a symmetric kernel) a convolution of the monomial $s^{q+1}$, of order $q+1$, with the kernel function $k(s)$ and $\tilde{v}$ is the same process as the one already treated, while $T$ is the monotone rearrangement map.

Thus let $x$ be a function satisfying the above assumption that it has $q$ vanishing derivatives at a point $t_{0}$ that is in the interior of its support, while $x^{q+1}\left(t_{0}\right)<0$. Suppose that $x_{n}$ is a preliminary 
estimator of $x$ that we partition as

$$
x(t)=x_{b, n}(t)+v_{n}(t) .
$$

Let $d_{n} \downarrow 0$ as $n \rightarrow \infty$, be a deterministic sequence. Define the rescaled deterministic and stochastic parts, respectively, by

$$
\begin{aligned}
g_{n}(s) & =d_{n}^{-(q+1)}\left(x_{b, n}\left(t_{0}+s d_{n}\right)-x\left(t_{0}\right)\right), \\
\tilde{v}_{n}\left(s ; t_{0}\right) & =d_{n}^{-(q+1)} v_{n}\left(t_{0}+s d_{n}\right),
\end{aligned}
$$

as in Section 3.2 and also

$$
\begin{aligned}
y_{n}(s) & =g_{n}(s)+\tilde{v}_{n}\left(s ; t_{0}\right) \\
& =d_{n}^{-(q+1)}\left(x_{n}\left(t_{0}+s d_{n}\right)-x\left(t_{0}\right)\right) .
\end{aligned}
$$

Then Assumption 1 is replaced by

Assumption 5. There exists a stochastic process $\tilde{v}\left(\cdot ; t_{0}\right) \neq 0$ such that

$$
\tilde{v}_{n}\left(\cdot ; t_{0}\right) \stackrel{\mathcal{L}}{\rightarrow} \tilde{v}\left(\cdot ; t_{0}\right)
$$

on $C(-\infty, \infty)$ as $n \rightarrow \infty$. The functions $\left\{x_{b, n}\right\}_{n \geq 1}$ are monotone and there exists a function $A(s)$ such that for each $c>0$

$$
\sup _{|s| \leq c}\left|g_{n}(s)-A(s)\right|
$$

as $n \rightarrow \infty$.

Let us also define the limit process

$$
y(s)=A(s)+\tilde{v}\left(s ; t_{0}\right) .
$$

Then we have the following general theorem.

Theorem 7. Let $J \subset \mathbb{R}$ be an interval, and $t_{0}$ a fixed point in the interior of $J$. Suppose that Assumption 5 holds. Assume furthermore that $\left\{y_{n}\right\}_{n \geq 1}$ and $y$ are continuous processes and that Assumption 2 holds for both $\left\{y_{n}\right\}_{n \geq 1}$ and $y$, defined in (40) and (41) respectively. Then

$$
d_{n}^{-(q+1)}\left[T_{J}\left(x_{n}\left(t_{0}\right)\right)-x\left(t_{0}\right)\right] \stackrel{\mathcal{L}}{\rightarrow} T\left[A(\cdot)+\tilde{v}\left(\cdot ; t_{0}\right)\right](0),
$$

as $n \rightarrow \infty$.

Proof. The proof is completely analogous to the proof of Theorem 4: At appropriate places, simply replace the factor $d_{n}$ with $d_{n}^{q+1}$ and $d_{n}^{-1}$ with $d_{n}^{-(q+1)}$. 


\subsection{Monotone density function estimation}

We now apply the above general result to the estimation of a monotone density $f$ function such that $f^{(j)}\left(t_{0}\right)=0$ for $j=1, \ldots, q$ and $f^{(q+1)}\left(t_{0}\right)<0$.

Recall the definition of the empirical distribution function $F_{n}$ and centered empirical distribution function $F_{n}^{0}$ in Section 3.3. Let $\delta_{n}$ be a sequence such that $\delta_{n} \downarrow 0, n \delta_{n} \uparrow \infty$ as $n \rightarrow \infty$, and define the centered empirical process $w_{n, \delta_{n}}$ locally around $t_{0}$ on the scale $\delta_{n}$, exactly as in Section 3.3, with the same definition of normalising factor $\sigma_{n, \delta_{n}}^{2}$. Furthermore, define the kernel function $x_{n}$ as the preliminary estimator of $f$ as in Section 3.3, and note that we can write the kernel estimator as $x_{n}=x_{b, n}+v_{n}$, with $x_{n}, x_{b, n}, v_{n}$ given in Equation (23) in Section 3.3. Again we will choose the bandwidth and the local scale to be the same $d_{n}=h$.

We now however obtain the rescaled process

$$
\tilde{v}_{n}\left(s ; t_{0}\right)=c_{n} \int k^{\prime}(u) w_{n, d_{n}}\left(s-u ; t_{0}\right) d u,
$$

with a (different) scale factor $c_{n}=d_{n}^{-(q+1)}(n h)^{-1} \sigma_{n, d_{n}}$.

Theorem 8. Let $\left\{t_{i}\right\}_{i \geq 1}$ be a stationary sequence with a monotone marginal density function $f$ such that

(i) $f^{(j)}\left(t_{0}\right)=0$ for $j=1, \ldots, q$,

(ii) $\sup _{t \in I_{t_{0}}} f^{(q+1)}(t)<0$,

(iii) $f \in C^{q+1}\left(I_{t_{0}}\right)$,

for an open interval $I_{t_{0}} \ni t_{0}$ where $t_{0}>0$. Assume that $\mathbb{E} t_{i}^{4}<\infty$. Let $x_{n}$ be the kernel density function defined above, with $k$ a bounded and compactly supported density such that $k^{\prime}$ is bounded. Suppose that one of the following conditions holds:

(a) $\left\{t_{i}\right\}_{i \geq 1}$ is an i.i.d. sequence,

(b) (1) $\left\{t_{i}\right\}_{i \geq 1}$ is a stationary $\phi$-mixing sequence with $\sum_{i=1}^{\infty} \phi^{1 / 2}(i)<\infty$;

(2) $f\left(t_{0}\right)=F^{\prime}\left(t_{0}\right)$ exists, as well as the joint density $f_{k}\left(s_{1}, s_{2}\right)$ of $\left(t_{1}, t_{1+k}\right)$ on $\left[t_{0}-\right.$ $\left.\delta, t_{0}+\delta\right]^{2}$ for some $\delta>0$, and $k \geq 1$;

(3) $\sum_{k=1}^{\infty} M_{k}<\infty$ holds, for $M_{k}=\sup _{t_{0}-\delta \leq s_{1}, s_{2} \leq t_{0}+\delta}\left|f_{k}\left(s_{1}, s_{2}\right)-f\left(s_{1}\right) f\left(s_{2}\right)\right|$.

Then choosing $h=a n^{-1 /(3+2 q)}$ and $a>0$ an arbitrary constant, we obtain

$$
n^{1 /(3+2 q)}\left\{\hat{f}_{n}\left(t_{0}\right)-f\left(t_{0}\right)\right\} \stackrel{\mathcal{L}}{\rightarrow} T\left(A_{f}(\cdot)+\tilde{v}_{n}\left(\cdot ; t_{0}\right)\right)(0),
$$

as $n \rightarrow \infty$, where $\tilde{v}(s ; t)$ is as in $(42)$, with $c=a^{-(q+3 / 2)} f\left(t_{0}\right)^{1 / 2}$, the function $A_{f}(s)$ is defined as

$$
A_{f}(s)=\frac{1}{(q+1) !} f^{(q+1)}\left(t_{0}\right) \int k(u)(s+u)^{q+1} d u
$$

and $w$ a standard two sided Brownian motion. 
Proof. The first part of Assumption 1 is established as in the proof of Theorem 5, with

$$
\tilde{v}\left(s ; t_{0}\right)=c \int k^{\prime}(u) w\left(s-u ; t_{0}\right) d u
$$

and $w$ a two sided standard Brownian motion, under the respective assumptions in (a) and (b).

Again we notice that $x_{b, n}(t)=h^{-1} \int k\left(\frac{t-u}{h}\right) f(u) d u$ is monotone. A change of variable and a Taylor expansion in the expression for $x_{b, n}$ proves the second part of Assumption 1 with limit function

$$
A(s)=\frac{1}{(q+1) !} f^{(q+1)}\left(t_{0}\right) \int k(u)(s+u)^{q+1} d u .
$$

To check Assumption 2 for the case $q \geq 1$ is much easier than for the case $q=0$, which is the case treated in Section 3. This can be seen in the statement of Lemma 1 in Anevski and Fougères [1]: the rescaled processes $\tilde{v}_{n}$ are the same for this new case $q \geq 1$ as for the already treated case $q=0$. However, the rescaled limit deterministic part $A(s)$ is, for $q \geq 1$, a smoothed out higher order monomial $(s+u)^{q+1}$, which of course has not linear increase/decrease (as when $r=0$ ) but polynomial increase. That means that one should compare $\tilde{v}_{n}$ in $A$ and $B$ of Lemma 1 not with for example, in $A$ of Lemma 1 the linear function $\tau(s-c)$ but instead with $\tau(s-c)^{q+1}$. But bounds of this form are easier to establish for polynomial increase than for linear increase, and in fact if we have established bounds for linear increase then we automatically get (at least) the same bounds for polynomial increase.

Finally in order to check that we can choose the scale $d_{n}$ so that $c_{n} \rightarrow c$, we make the following calculations.

(1) Independent data case (a): We have $\sigma_{n, d_{n}}^{2} \sim n d_{n} f\left(t_{0}\right)$, so that

$$
\begin{aligned}
c_{n} & =d_{n}^{-(q+1)}(n h)^{-1} \sigma_{n, d_{n}} \\
& \sim d_{n}^{-(q+3 / 2)} n^{-1 / 2} f\left(t_{0}\right)^{1 / 2} .
\end{aligned}
$$

This tells us that we should choose $d_{n}=a n^{-1 /(3+2 q)}$ we get $c=a^{-3 / 2} f\left(t_{0}\right)^{1 / 2}$.

(2) Mixing data case (b): Similar to the proof of case (a).

\subsection{Monotone regression function estimation}

Next, we treat the regression estimation problem for a regression function $m$ such that $m^{(j)}\left(t_{0}\right)=$ for $j=1, \ldots, q$ and $m^{(q)}\left(t_{0}\right)<0$ for $t_{0}$ the point of interest. We have the same setting for the regression problem as in Section 3.4, so $m$ is a function defined on [0,1], equidistant design $t_{i}=i / n$, and data $\left(t_{i}, y_{i}\right)$ from the model $y_{i}=m\left(t_{i}\right)+\varepsilon_{i}$ where $\left\{\varepsilon_{i}\right\}$ is a stationary sequence of independent, weakly dependent or long range dependent data. Define the two-sided partial sum process $w_{n}$ and the function $\bar{y}_{n}$ exactly as in Section 3.4, and the Gasser-Müller kernel estimate as in Equation (26). The monotone estimator of $m$ is defined as $\tilde{m}(t)=T_{[0,1]}\left(x_{n}\right)(t)$. Again we can write $x_{n}=x_{b, n}+v_{n}$, with $x_{b, n}$ and $v_{n}$ given in Equation (28). We note that since the process 
$w_{n}$ is defined exactly the same way as in Section 3.4, we have that $w_{n}$ converges to a limit process which is Brownian motion or Fractional Brownian motion, or something else, depending on the dependence structure for the data $\left\{\varepsilon_{i}\right\}$, as in Section 3.4.

We choose bandwidth equal to the local scale factor $h=d_{n}$, and rescale the process part as

$$
\tilde{v}_{n}=d_{n}^{-(q+1)}(n h)^{-1} \sigma_{\hat{n}} \int \bar{w}_{\hat{n}}(s-u) k^{\prime}(u) d u,
$$

while the deterministic part is rescaled as

$$
g_{n}(s)=d_{n}^{-(q+1)}\left(x_{b, n}\left(t_{0}+s d_{n}\right)-x\left(t_{0}\right)\right) .
$$

Theorem 9. Assume $m$ is monotone on $[0,1]$ and

(i) $m^{(j)}\left(t_{0}\right)=0$ for $j=1, \ldots, q$,

(ii) $\sup _{t \in I_{t_{0}}} m^{(q+1)}(t)<0$,

(iii) $m \in C^{q+1}\left(I_{t_{0}}\right)$,

for an open interval $I_{t_{0}} \ni t_{0}$, with $t_{0} \in(0,1)$. Let $x_{n}$ be the kernel estimate of $m$ defined above, with a non-negative and compactly supported kernel $k$ such that $k^{\prime}$ is bounded, and with bandwidth $h$ specified below. Suppose that one of the following conditions holds.

(a) $\left\{\varepsilon_{i}\right\}$ are independent and identically distributed, $E \varepsilon_{i}=0 ; \sigma^{2}=\operatorname{Var}\left(\varepsilon_{i}\right)<\infty$, and $h=$ $a n^{-1 /(3+2 q)}$, for an arbitrary $a>0$,

(b) Assumption 3 or 4 holds, $\sigma_{n}^{2}=\operatorname{Var}\left(\sum_{i=1}^{n} \varepsilon_{i}\right), \kappa^{2}$ is defined in (30), $h=a n^{-1 /(3+2 q)}$, with $a>0$ an arbitrary constant,

(c) $\varepsilon_{i}=g\left(\xi_{i}\right)$ is a long range dependent subordinated Gaussian sequence with parameters $d$ and $r, h=l_{2}(n ; a) n^{-r d /(2+q+r d)}$ with $a>0$ and $n \mapsto l_{2}(n ; a)$ is a slowly varying function defined in the proof below.

Then, correspondingly, we obtain

$$
d_{n}^{-1}\left\{\tilde{m}\left(t_{0}\right)-m\left(t_{0}\right)\right\} \stackrel{\mathcal{L}}{\rightarrow} T\left[A_{m}(\cdot)+\tilde{v}\left(\cdot ; t_{0}\right)\right](0),
$$

as $n \rightarrow \infty$, where

$$
\begin{aligned}
& \tilde{v}(s ; t)=c \int w(s-u) k^{\prime}(u) d u, \\
& A_{m}(s)=\frac{m^{(q+1)}\left(t_{0}\right)}{(q+1) !} \int(u+s)^{q+1} k(u) d u
\end{aligned}
$$

and respectively

(a) $w=B ; c=\sigma a^{-(q+3 / 2)}$,

(b) $w=B ; c=\kappa a^{-(q+3 / 2)}$,

(c) $w=B_{r, \beta} ; c=\left|\eta_{r}\right| a$ (where $\beta$ defined in (32)). 
Proof. If we choose $d_{n}=h$ in such a way so that

$$
d_{n}^{-(q+1)}(n h)^{-1} \sigma_{\hat{n}} \rightarrow c>0,
$$

then we obtain the first part of Assumption 1

$$
\tilde{v}_{n}(s ; t) \stackrel{\mathcal{L}}{\rightarrow} \tilde{v}(s ; t)
$$

as in the proof of Theorem 6.

For the rescaling of the deterministic part $g_{n}$, similarly to as in the proofs of Theorem 6 and Theorem 8, one can show that the second part of Assumption 1 holds, with

$$
A_{m}(s)=\frac{m^{(q+1)}\left(t_{0}\right)}{(q+1) !} \int(u+s)^{q+1} k(u) d u .
$$

The proof that Assumption 2 holds is similar to the reasoning in the proof of Theorem 8 .

It only remains to check whether $d_{n}^{-1}(n h)^{-1} \sigma_{\hat{n}} \rightarrow c>0$ for the three types of dependence.

1. Independent case (a): We have $\sigma_{\hat{n}}^{2}=\sigma^{2} n d_{n}$. Thus $d_{n}^{-(q+1)}(n h)^{-1} \sigma_{\hat{n}}=\sigma n^{-1 / 2} d_{n}^{-(q+3 / 2)}$, and (45) is satisfied with $c=\sigma a^{-(q+3 / 2)}$ if $d_{n}=h=a n^{-1 /(3+2 q)}$.

2. Mixing case (b): The proof is similar to the proof of (a), replacing $\sigma$ by $\kappa$.

3. Long range data case (c): Since $\sigma_{\hat{n}}^{2}=\eta_{r}^{2}\left(n d_{n}\right)^{2-r d} l_{1}\left(n d_{n}\right)$, if we choose $d_{n}=h$ we will have

$$
\begin{aligned}
d_{n}^{-(q+1)}(n h)^{-1} \sigma_{\hat{n}} & =d_{n}^{-(q+1)}(n h)^{-1}\left|\eta_{r}\right|\left(n d_{n}\right)^{1-r d / 2} l_{1}\left(n d_{n}\right)^{1 / 2} \\
& \rightarrow\left|\eta_{r}\right| a
\end{aligned}
$$

if and only if

$$
d_{n}=n^{-r d /(q+2+r d)} l_{2}(n ; a),
$$

where $l_{2}$ is another function slowly varying at infinity, implicitly defined in (46). Thus, (45) follows with $c=\left|\eta_{r}\right| a$ and $h=d_{n}$ given in (47).

\section{Conclusions}

We considered the feature of estimating an arbitrary monotone function $x$, via a monotone rearrangement of a "preliminary" estimator $x_{n}$ of the unknown $x$. We derived consistency and limit distribution results for the monotonized estimator that hold under rather general dependence assumptions.

The work done here has been with the use of kernel based methods for the preliminary estimator $x_{n}$ of $x$. Other methods, such as wavelet based ones, are possible. We would like to emphasise that the only assumptions required are given in Assumptions 1 and 2. 
The small simulation study that has been performed in Section 2 of Anevski and Fougères [1] deals with independent data, as also done in Dette et al. [13] and Birke and Dette [6]. This independence framework is the only one considered in the density context too. A larger panel of dependence situations in the comparisons would clearly be of interest.

We have studied applications to density and regression function estimation. Other estimation problems that are potentially possible to treat with our methods are for example, spectral density estimation, considered by Anevski and Soulier [3], and deconvolution, previously studied by van Es et al. [37].

\section{Appendix A: Proofs of Section 2}

Proof of Lemma 1. Assertions (i) and (ii) both follow from the fact that

$$
\begin{aligned}
\lim _{u \rightarrow u_{0}}\left|r_{f, I}(u)-r_{f, I}\left(u_{0}\right)\right| & =\lim _{u \rightarrow u_{0}} \lambda\left\{t \in I: \max \left(u, u_{0}\right) \geq f(t)>\min \left(u, u_{0}\right)\right\} \\
& =\lambda\left\{I \cap f^{-1}\left(\left\{u_{0}\right\}\right)\right\},
\end{aligned}
$$

which is equal to 0 in (i), and to $c$ in (ii). Finally, assertion (iii) arises from writing that $r_{f, I}(u)=$ $r_{f, I}\left(f\left(t_{0}^{-}\right)\right)=t_{0}$ for each $u \in\left(f\left(t_{0}^{+}\right), f\left(t_{0}^{-}\right)\right)$.

Proof of Lemma 2. (i)-(iii) follow from the definition; indeed, for each $u \in f(I)+c$, $r_{f+c, I}(u)=\lambda\{t \in I: f(t)+c>u\}=r_{f, I}(u-c)$, and for each $u \in c f(I), r_{c f, I}(u)=\lambda\{t \in$ $I: c f(t)>u\}=r_{f, I}(u / c)$ if $c>0$. As for (iii), $\{t \in I: f(t)>u\} \subset\{t \in I: g(t)>u\}$, for each fixed $u$, if $f \leq g$. Statement (iv) follows from $r_{f_{c}, I}(u)=\lambda\{t \in I / c: f(c t)>u\}=$ $\lambda\{s / c \in I / c: f(s)>u\}=r_{f, I}(u) / c$, for each $u \in f(I)$. Statement (v) is a consequence of $r_{f_{c}, I}(u)=\lambda\{t \in I-c: f(t+c)>u\}=\lambda\{s-c \in I-c: f(s)>u\}=\lambda\{t \in I: f(t)>u\}$, for each $u \in f(I)$.

Proof of Lemma 3. Let $I=[a, b]$; each assertion is a consequence of its counterpart in Lemma 2. Let $t \in I$; statement (i) follows from $T_{I}(f+c)(t)=\inf \left\{u \in f(I)+c: r_{f, I}(u-c) \leq\right.$ $t-a\}=T_{I}(f)(t)+c$, whereas (ii) comes from $T_{I}(c f)(t)=\inf \left\{u \in c f(I): r_{f, I}(u / c) \leq t-a\right\}=$ $c T_{I}(f)(t)$. To show (iii), note that $f \leq g \Rightarrow r_{f, I} \leq r_{g, I} \Rightarrow T_{I}(f) \leq T_{I}(g)$. Assertion (iv) follows from the fact that for each $t \in I / c, T_{I / c}\left(f_{c}\right)(t)=\inf \left\{u \in f(I): r_{f, I}(u) \leq c t-a\right\}=T_{I}(f)(c t)$. Finally, statement $(\mathrm{v})$ follows since for each $t \in I-c, T_{I-c}\left(f_{c}\right)(t)=\inf \left\{u \in f(I): r_{f, I}(u) \leq\right.$ $t+c-a\}=T_{I}(f)(t+c)$.

Proof of Theorem 1. Let $f, g$ be functions in $\mathcal{B}(I)$. Clearly $g(u)-\|f-g\| \leq f(u) \leq$ $g(u)+\|f-g\|$, which by Lemma 3(i) and (iii) implies that $T_{I}(g)(u)-\|f-g\| \leq T_{I}(f)(u) \leq$ $T_{I}(g)(u)+\|f-g\|$, so that $\left|T_{I}(f)-T_{I}(g)\right|(u) \leq\|f-g\|$, for each $u$. Since the right hand side is independent of $u$, the absolute value on the left hand side can be replaced by the supremum norm, which implies the statement of the theorem. 


\section{Appendix B: Maximal bounds for rescaled partial sum and empirical processes}

In this section, we state conditions under which Assumption 2 holds. Further specialisations of these conditions to density and regression function estimation are given in the supplemental article (Anevski and Fougères [1]).

Recall that

$$
\begin{aligned}
& \tilde{g}_{n}(s)=d_{n}^{-1}\left\{x_{b, n}\left(t_{0}+s d_{n}\right)-x_{b, n}\left(t_{0}\right)\right\} \\
& \tilde{v}_{n}(s)=d_{n}^{-1} v_{n}\left(t_{0}+s d_{n}\right) .
\end{aligned}
$$

Since under Assumption 1

$$
\begin{aligned}
y_{n}(s)-\left\{\tilde{g}_{n}(s)+\tilde{v}_{n}(s)\right\} & =d_{n}^{-1}\left\{x_{b, n}\left(t_{0}\right)-x\left(t_{0}\right)\right\} \\
& \rightarrow \Delta,
\end{aligned}
$$

as $n \rightarrow \infty$, and $|\Delta|<\infty$, establishing Assumption 2 for the process $\tilde{g}_{n}+\tilde{v}_{n}$ implies that it holds also for the process $y_{n}=g_{n}+\tilde{v}_{n}$. Therefore, it is enough to establish Assumption 2 for $y_{n}$ replaced by $\tilde{g}_{n}+\tilde{v}_{n}$.

Recall that for the cases that we cover the rescaled processes are of the form

$$
\tilde{v}_{n}\left(s ; t_{0}\right)=c_{n} \int k^{\prime}(u) z_{n}\left(s-u ; t_{0}\right) d u
$$

with $z_{n}=w_{n, d_{n}}$ the local rescaled empirical process in the density estimation case and $z_{n}=w_{n}$ the partial sum process in the regression case. This implies that for the density estimation case the support of $\tilde{v}_{n}$ is stochastic, since it depends on $\max _{1 \leq i \leq n} t_{i}$, while for the regression estimation case it does not depend on the data $\left\{\varepsilon_{i}\right\}$ and is as a matter of fact compact and deterministic.

The proof of the following Lemma is given in the supplemental article (Anevski and Fougères [1]).

Lemma 6. Let $\operatorname{supp}(k) \subset[-1,1]$. Suppose that Assumption 1 holds. Assume that $t_{0}$ has a neighbourhood $I=\left[t_{0}-\varepsilon, t_{0}+\varepsilon\right]$ such that $\tau:=\sup _{t \in I} x^{\prime}(t)<0$. Suppose also that

$$
x_{b, n}^{\prime}\left(t+s d_{n}\right) \rightarrow x^{\prime}(t)
$$

as $n \rightarrow \infty$, for all $t \in I$.

Then (14) and (15) written for $z_{n}=\tilde{g}_{n}+\tilde{v}_{n}$ are implied by the two results:

(A) For every $\delta>0$ and $0<c<\infty$ there is a finite $M>0$

$$
\liminf _{n \rightarrow \infty} P\left[\bigcap_{s \in\left(c, d_{n}^{-1} \varepsilon\right)}\left\{\tilde{v}_{n}(s)<\frac{M}{2}-\tau(s-c)\right\}\right]>1-\delta .
$$


(B) For every $\delta>0$ and finite $M>0$ there is a finite $C$, not depending on $\delta$, such that for each $c>C$

$$
\limsup _{n \rightarrow \infty} P\left\{\sup _{s \in d_{n}^{-1}(0, \ell(n))} \tilde{v}_{n}(s)>\frac{M}{2}-\tau\left(d_{n}^{-1} \varepsilon-c\right)\right\}<\delta,
$$

where $\ell(n)$ is a deterministic function which satisfies either of

(i) $\liminf _{n \rightarrow \infty} P\left\{\max _{1 \leq i \leq n} t_{i}<\ell(n)\right\}=1$,

or

(ii) $\ell(n) \equiv \max \operatorname{supp}\left(x_{n}\right)$ if $\lim \sup _{n \rightarrow \infty} \max \operatorname{supp}\left(x_{n}\right) \leq K<\infty$.

\title{
Acknowledgements
}

We thank Philippe Soulier for valuable discussions during numerous occasions, and in particular for pointing out the simple conditions for Lemma 4. The second author is also grateful to Philippe Barbe who provided several precious suggestions for paper [16]. Both authors would like to thank the referee of a previous version of the paper, for pointing out several deficiencies therein. We would also like to thank an Associate Editor and two referees for the many valuable comments, and in particular for enquiring about the $q$ vanishing derivatives case, which has given fruit to the results in Section 4. A special thank also to Mrs Stenshuvud for providing inspiration.

\section{Supplementary Material}

\begin{abstract}
Supplement to "Limit properties of the monotone rearrangement for density and regression function estimation” (DOI: 10.3150/17-BEJ998SUPP; .pdf). The supplemental article Anevski and Fougères [1] provides in a first section some technical results on maximal bounds for the rescaled version partial sum and empirical process; it gives further conditions under which Assumption 2 holds, with application to the density and regression function estimation cases, stated in Appendix B, as well as all proofs. Furthermore, Section 2 of Anevski and Fougères [1] contains a simulation study that illustrates the finite sample behaviour of our estimator, and compare it to other estimators that are considered in the paper of Birke and Dette [6].
\end{abstract}

\section{References}

[1] Anevski, D. and Fougères, A.-L. (2017). Supplement to "Limit properties of the monotone rearrangement for density and regression function estimation.” DOI:10.3150/17-BEJ998SUPP.

[2] Anevski, D. and Hössjer, O. (2006). A general asymptotic scheme for inference under order restrictions. Ann. Statist. 34 1874-1930. MR2283721

[3] Anevski, D. and Soulier, P. (2011). Monotone spectral density estimation. Ann. Statist. 39 418-438. MR2797852

[4] Billingsley, P. (1968). Convergence of Probability Measures. New York: Wiley. 
[5] Birke, M. and Dette, H. (2007). Estimating a convex function in nonparametric regression. Scand. J. Stat. 34 384-404. MR2346646

[6] Birke, M. and Dette, H. (2008). A note on estimating a smooth monotone regression by combining kernel and density estimates. J. Nonparametr. Stat. 20 679-691.

[7] Birke, M., Neumeyer, N. and Volgushev, S. (2013). The independence process in conditional quantile location-scale models and an application to testing for monotonicity. Submitted.

[8] Birke, M., Neumeyer, N. and Volgushev, S. (2016). The independence process in conditional quantile location-scale models and an application to testing for monotonicity. Available at arXiv:1609.07696.

[9] Brunk, H.D. (1958). On the estimation of parameters restricted by inequalities. Ann. Math. Stat. 29 437-454.

[10] Chernozhukov, V., Fernandez-Val, I. and Galichon, A. (2007). Improving point and interval estimates of monotone functions by rearrangement. Biometrika 96 559-575.

[11] Chernozhukov, V., Fernandez-Val, I. and Galichon, A. (2010). Quantile and probability curves without crossing. Econometrica 78 1093-1125.

[12] Csőrgő, S. and Mielniczuk, J. (1995). Density estimation under long-range dependence. Ann. Statist. 23 990-999.

[13] Dette, H., Neumeyer, N. and Pilz, K.F. (2006). A simple nonparametric estimator of a strictly monotone regression function. Bernoulli 12 469-490.

[14] Dette, H. and Pilz, K.F. (2006). A comparative study of monotone nonparametric kernel estimates. J. Stat. Comput. Simul. 76 41-56. MR2225147

[15] Eggermont, P.P.B. and LaRiccia, V.N. (2000). Maximum likelihood estimation of smooth monotone and unimodal densities. Ann. Statist. 28 922-947. MR1792794

[16] Fougères, A.-L. (1997). Estimation de densités unimodales. Canad. J. Statist. 25 375-387.

[17] Gasser, T. and Muller, H.-G. (1984). Estimating regression functions and their derivatives by the kernel method. Scand. J. Stat. 11 171-185.

[18] Gijbels, I. (2004). Monotone regression. Discussion paper No. 0334. Institut de Statistique, Université Catholique de Louvain.

[19] Grenander, U. (1956). On the theory of mortality measurements. Part II. Skand. Aktuarietidskr. 39.

[20] Hall, P. and Huang, L.-S. (2001). Nonparametric kernel regression subject to monotonicity constraints. Ann. Statist. 29 624-647. MR1865334

[21] Hall, P. and Kang, K.-H. (2005). Unimodal kernel density estimation by data sharpening. Statist. Sinica 15 73-98.

[22] Hardy, G.H., Littlewood, J.E. and Pólya, G. (1952). Inequalities, 2nd ed. Cambridge: Cambridge University Press.

[23] Jankowski, H.K. and Wellner, J.A. (2009). Estimation of a discrete monotone distribution. Electron. J. Stat. 3 1567-1605. MR2578839

[24] Lieb, E.H. and Loss, M. (1996). Analysis. Graduate Studies in Mathematics 14. Providence, RI: Amer. Math. Soc.

[25] Mammen, E. (1991). Nonparametric regression under qualitative smoothness assumptions. Ann. Statist. 19 741-759. MR1105842

[26] Mammen, E., Marron, J.S., Turlach, B.A. and Wand, M.P. (2001). A general projection framework for constrained smoothing. Statist. Sci. 16 232-248.

[27] Meyer, M.C. and Woodroofe, M. (2004). Consistent maximum likelihood estimation of a unimodal density using shape restrictions. Canad. J. Statist. 32 85-100.

[28] Mukerjee, H. (1988). Monotone nonparametric regression. Ann. Statist. 16 741-750.

[29] Neumeyer, N. (2007). A note on uniform consistency of monotone function estimators. Statist. Probab. Lett. 77 693-703. 
[30] Polonik, W. (1995). Density estimation under qualitative assumptions in higher dimensions. J. Multivariate Anal. 55 61-81.

[31] Polonik, W. (1998). The silhouette, concentration functions and ML-density estimation under order restrictions. Ann. Statist. 26 1857-1877. MR1673281

[32] Prakasa Rao, B.L.S. (1969). Estimation of a unimodal density. Sankhya, Ser. A 31 23-36.

[33] Ramsay, J.O. (1988). Monotone regression splines in action (with comments). Statist. Sci. 3 425-461.

[34] Schwartz, L. (1966). Théorie des Distributions. Publications de l'Institut de Mathématique de l'Université de Strasbourg, No. IX-X. Nouvelle Édition, Entiérement Corrigée, Refondue et Augmentée. Paris: Hermann. MR0209834

[35] Taqqu, M.S. (1974/75). Weak convergence to fractional Brownian motion and to the Rosenblatt process. Z. Wahrsch. Verw. Gebiete 31 287-302. MR0400329

[36] Taqqu, M.S. (1979). Convergence of integrated processes of arbitrary Hermite rank. Z. Wahrsch. Verw. Gebiete 50 53-83.

[37] van Es, B., Jongbloed, G. and van Zuijlen, M. (1998). Isotonic inverse estimators for nonparametric deconvolution. Ann. Statist. 26 2395-2406.

[38] van der Vaart, A.W. and van der Laan, M.J. (2003). Smooth estimation of a monotone density. Statistics 37 189-203.

[39] Villani, C. (2003). Topics in Optimal Transportation. Graduate Studies in Mathematics 58. Providence, RI: Amer. Math. Soc.

[40] Volguchev, S. (2013). Smoothed quantile regression processes for binary response models. Available at arXiv: 1302.5644 .

[41] Volguchev, S., Birke, M., Dette, H. and Neumeyer, N. (2013). Significance testing in quantile regression. Electron. J. Stat. 7 105-145.

[42] Volgushev, S. and Dette, H. (2013). Nonparametric quantile regression for twice censored data. Bernoulli 19 748-779.

[43] Wand, M.P. and Jones, M.C. (1995). Kernel Smoothing. Monographs on Statistics and Applied Probability 60. London: Chapman \& Hall. MR1319818

[44] Wright, F.T. (1981). The asymptotic behaviour of monotone regression estimates. Ann. Statist. $9443-$ 448.

Received August 2016 and revised October 2017 